This item was submitted to Loughborough's Research Repository by the author.

Items in Figshare are protected by copyright, with all rights reserved, unless otherwise indicated.

\title{
A generalized model of advertised sales
}

PLEASE CITE THE PUBLISHED VERSION

https://doi.org/10.1257/mic.20170152

PUBLISHER

American Economic Association

VERSION

VoR (Version of Record)

\section{PUBLISHER STATEMENT}

The author has the right to republish, post on servers, redistribute to lists and use any component of this work in other works. For others to do so requires prior specific permission and/or a fee. Permissions may be requested from the American Economic Association Administrative Office.

\section{LICENCE}

CC BY-NC-ND 4.0

\section{REPOSITORY RECORD}

Shelegia, Sandro, and Christopher Wilson. 2021. "A Generalized Model of Advertised Sales”. Loughborough University. https://hdl.handle.net/2134/12241229.v1. 


\title{
A Generalized Model of Advertised Sales ${ }^{\dagger}$
}

\author{
By SANDro Shelegia ANd Chris M. Wilson*
}

\begin{abstract}
To better understand temporary price reductions or "sales," this paper presents a generalized "clearinghouse" framework of advertised sales and explores some applications. By viewing the firms as competing in utility and amending the conventional tiebreak rule, we allow for multiple dimensions of firm heterogeneity in complex market environments. Moreover, we (i) provide original insights into the number and types of firms that use sales, (ii) offer new results on how firm heterogeneity affects market outcomes, (iii) extend a common empirical "cleaning" procedure, and (iv) analyze a family of activities in sales markets, including persuasive advertising and obfuscation. (JEL D21, D43, L13, L25, M37)
\end{abstract}

T he evidence of price dispersion within markets is overwhelming, even when products are homogeneous (as reviewed by Baye, Morgan, and Scholten 2006). Such price dispersion has a long-standing interest within the areas of industrial organization and marketing, but it is also gaining increased attention from finance, development economics, and macroeconomics. ${ }^{1}$ Empirical findings show that much price dispersion is due to temporary price reductions or "sales" (e.g., Hosken and Reiffen 2004; Nakamura and Steinsson 2008; Kaplan and Menzio 2015; Hitsch, Hortacsu, and Lin 2019). One of the main theoretical explanations for such sales involves mixed strategies that arise from variation in consumers' search frictions and/or the existence of moderate advertising costs ${ }^{2}$ This literature continues to offer deep insights into sales and is also receiving a renewed interest as an analytical foundation

\footnotetext{
* Shelegia: Department of Economics and Business, Universitat Pompeu Fabra, Ramon Trias Fargas 25-27, Barcelona 08005, and Barcelona GSE (email: sandro.shelegia@upf.edu); Wilson: School of Business and Economics, Loughborough University, Loughborough LE11 3TU, United Kingdom (email: c.m.wilson@lboro. ac.uk). Robert Porter was coeditor for this article. We thank the referees. We also thank Mark Armstrong, Mike Baye, Andrew Rhodes, Yossi Spiegel, Matthijs Wildenbeest, and various seminar participants. A previous version of the paper was titled, "A Generalized Model of Sales." Shelegia acknowledges financial support from the Spanish Ministry of Economy and Competitiveness through the Severo Ochoa Programme for Centres of Excellence in R\&D SEV-2015-0563, and the research grants RYC-2016-20307 and ECO2017- 89240-P.

Go to https://doi.org/10.1257/mic.20170152 to visit the article page for additional materials and author disclosure statement(s) or to comment in the online discussion forum.

${ }^{1}$ For example, see, respectively, Woodward and Hall (2012); Allen, Clark, and Houde (2014); Jensen (2007); Nakamura et al. (2018).

${ }^{2}$ For example, see, respectively, Varian (1980), Burdett and Judd (1983), Stahl (1989), Janssen and MoragaGonzález (2004), Robert and Stahl (1993), Baye and Morgan (2001). See Baye, Morgan, and Scholten (2006) and Anderson and Renault (2018) for reviews.
} 
for many broader research areas, including price comparison platforms, advertising, obfuscation, choice complexity, and several issues within macroeconomics. ${ }^{3}$

However, while the predictions of such mixed strategy sales models are frequently consistent with empirical evidence, they often struggle to fully explain the observed differences in $\triangle$ rms $\bigotimes$ pricing and advertising behaviors. ${ }^{4}$ In particular, their restricted ability to allow for $\triangle \mathrm{rm}$ heterogeneity constrains the theoretical and empirical understanding of sales and inhibits the wider related literatures. Indeed, as Baye and Morgan $(2009,1151)$ state, $\mathbb{X}$ little is known about asymmetric models within this class. Breakthroughs on this front would not only constitute a major theoretical advance, but permit a tighter $\nabla t$ between the underlying theory and empirics. $\square$

In response, this paper presents a substantially generalized $\bigotimes$ clearinghouse区 framework of advertised sales (e.g., Baye and Morgan 2001; Baye, Morgan, and Scholten 2004a) and explores some example applications. Our main contribution is methodological $\bigotimes$ while its modeling assumptions sometimes differ to existing research, our framework can extend many of the past literature $\$$ sales predictions to more complex market settings while allowing for multiple dimensions of $\nabla r m$ heterogeneity. Thus, we hope that our framework will help open up the future analysis of many remaining questions within the sales literature. As some examples, the paper then provides original insights into the number and type of 冈rms that use sales, offers new predictions about the effects of $\nabla$ rm asymmetries on market outcomes, and illustrates how the framework can be used to (i) extend a $₫$ cleaning $\square$ procedure that is commonly used in the empirical literature, and (ii) đanalyze a family of activities in sales markets, including persuasive advertising and obfuscation.

As reviewed by Baye, Morgan, and Scholten (2006), the original clearinghouse framework considers a symmetric market with a single homogeneous good. Consumers are potentially split into \nonshoppers, $₫$ who are only willing to buy from a designated $\bigotimes \mathrm{rm}$, and $\bigotimes$ shoppers,$\nabla$ who can buy from any $\nabla \mathrm{rm}$. Firms choose their price and whether to inform consumers of this price via some advertising channel. As consistent with observed sales behavior, the equilibrium involves each $\nabla r m$ randomizing between selecting a high price without advertising, and advertising a lower price drawn from some support. The seminal model of sales by Varian (1980) obtains as the limiting case when advertising costs tend to zero.

We modify this clearinghouse framework in two important respects. First, we recast the $\nabla r m s$ as competing in (net) utility rather than prices. By drawing on the (symmetric, pure strategy) model of competition in the utility space by Armstrong and Vickers (2001), we let each $\bigotimes \mathrm{rm} i$ select its utility, $u_{i}$, with a per-consumer pro®t function, $\pi_{i}\left(u_{i}\right)$, that depends upon the $\bigotimes$ rm $\varangle$ underlying demand, products, costs, and pricing technology. With little increase in computation, this facilitates a high

\footnotetext{
${ }^{3}$ For reviews and recent examples, see Moraga-Gonz囚lez and Wildenbeest (2012), Armstrong (2015), Spiegler (2016), and Ronayne (2020). For macroeconomic applications to nominal rigidities, output \uctuations, and monetary policy, see Guimaraes and Sheedy (2011), Kaplan and Menzio (2016), and Burdett and Menzio (2018).

${ }^{4}$ For example, Lach (2002); Baye, Morgan, and Scholten (2004a, 2004b); Lewis (2008); Chandra and Tappata (2011); Wildenbeest (2011); Giulietti, Waterson, and Wildenbeest (2014); Lach and Moraga-Gonz囚lez (2017); Zhang, Chan, and Xie (2018); and Pennerstorfer et (2020). Also see Potters and Suetens (2013) for experimental evidence.
} 
level of generality across complex market settings, involving downward-sloping demand, multiple products, or two-part tariffs, where the analysis of sales would often be otherwise impenetrable.

Second, we make a subtle change to the tiebreak rule when the shoppers are indifferent over which $\triangle \mathrm{rm}$ to trade with. The existing literature assumes shoppers (i) trade exclusively with advertising $\nabla \mathrm{rm}(\mathrm{s})$ in any tie between advertising and nonadvertising $\bigotimes$ rms, and (ii) mix between the tied $\triangle \mathrm{rms}$ with equal probability in any other form of tie. Although consistent with an advertising channel involving a price comparison platform where shoppers face additional visit costs to buy from

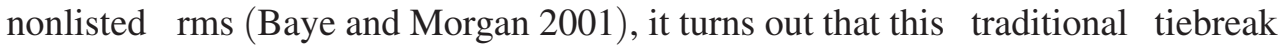
rule impedes the analysis of sales under $\bigotimes$ rm heterogeneity. In particular, it causes a substantial loss in tractability by (i) making the exact form of equilibrium become dependent upon parameter subcases, and (ii) prompting the existence of mass points in some $₫ \mathrm{rms} \bigotimes$ advertised price distributions. ${ }^{5}$

To resolve this problem, we take a different approach within a setting where shoppers receive all adverts before making their visit decisions. Here, we are free to select any tiebreak rule because shoppers should be willing to buy from any advertising or nonadvertising $\bigotimes$ rm with the same expected utility. Moreover, rather than treating the tiebreak rule as a modeling assumption, we are free to specify the tiebreak rule as part of equilibrium in line with Simon and Zame区 (1990) concept of \endogenous sharing rules $\$ (which they use to offer general results about equilibrium existence in $n$-player games). Under our assumptions, we show that the

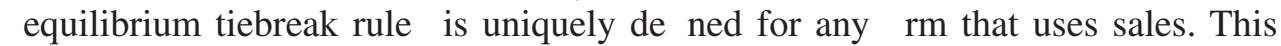
rule partially offsets any $\nabla r m$ heterogeneities and ensures that all $\nabla r m s$ that use sales have the same incentive to employ a common upper bound in advertised utilities. In symmetric settings, it coincides with the traditional tiebreak rule. However, in asymmetric settings, it offers a unique level of tractability by removing any parameter subcases and eliminating any mass points in advertised utility distributions. As such, it allows us to simultaneously permit (i) any variation in $\bigotimes$ rms $\bigotimes$ shares of nonshoppers, (ii) any variation in $₫$ rms $\bigotimes$ advertising costs, and (iii) considerable variation in $₫ \mathrm{rms} \bigotimes$ pro®t functions. In equilibrium, rms may vary in advertising probabilities, utility distributions, and pro囚ts depending on the level and form of heterogeneity. ${ }^{6}$

Sections I and II introduce the framework and equilibrium analysis. We Ørst present the equilibrium under duopoly and show how it can generalize many predictions from the previous literature to more complex market settings with multiple forms of heterogeneity. $]^{7}$ In addition, we offer further new insights by characterizing some

\footnotetext{
${ }^{5}$ For instance, even in a simple unit demand duopoly where \rms only differ in their shares of nonshoppers, the equilibrium in Arnold et 1 . (2011) has two parameter subcases and one \rm uses a mass point in advertised prices. Furthermore, the equilibrium does not converge to standard sales equilibria as advertising costs tend to zero (e.g., Narasimhan 1988).

${ }^{6}$ The variation in pro囚t functions is subject to a condition that is implicit within all of the existing literature区

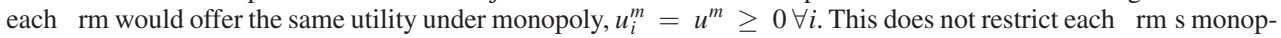
oly pro囚ts and is innocuous in several market settings, including unit demand.

${ }^{7}$ Among many others, these include symmetric models such as Varian (1980); Simester (1997); Baye, Morgan, and Scholten (2004a, 2006); and asymmetric models, such as Narasimhan (1988); Baye, Kovenock, and de Vries (1992); Kocas and Kiyak (2006); and Wildenbeest (2011).
} 
common forms of sales that have remained unstudied within the clearinghouse literature, including cases where $\triangle$ rms use two-part tariffs or nonprice variables such as package size (e.g., $₫ X \%$ Free区). We then present the equilibrium for $n>2 \bigotimes$ rms.

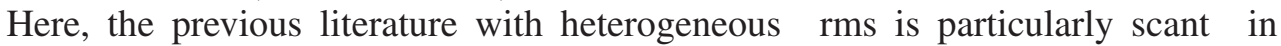
a simple setting of unit demand and zero advertising costs, it suggests that only two 『rms can ever engage in sales behavior (Baye, Kovenock, and de Vries 1992; Kocas and Kiyak 2006; and Shelegia 2012). In contrast, and in better line with typical empirical Øndings (e.g., Lach 2002, Lewis 2008, Chandra and Tappata 2011), our framework explains how any number of heterogeneous $\operatorname{rrms}^{*} \in[2, n]$ can engage in equilibrium sales. In particular, we demonstrate how a rise in the cost of informative advertising can lead to an increase in the number of $\nabla \mathrm{rms}$ that use advertised sales. Intuitively, despite the direct cost increase, higher advertising costs can prompt more $\triangle$ rms to use sales by softening competition for the shoppers. Thus, if the costs of informative advertising fall in the digital era, we predict that fewer Ørms will engage in sales behavior. Finally, we provide a broad characterization of the types of $\nabla r m s$ that are most likely to use advertised sales. Ceteris paribus, these are $\bigotimes$ rms with relatively low shares of loyal consumers, low advertising costs, and high pro囚tability (under reasonable assumptions on market conditions). These results offer some clear empirical predictions, but they currently remain untested within the literature. ${ }^{8}$

SectionXII explores some applications to illustrate how our framework can be used for future research. SectionХIIA uses the framework to assess and extend a common procedure within the large empirical literature on sales and price dispersion. This $₫ \mathrm{cleaning} \bigotimes$ procedure attempts to remove the effects of $\nabla \mathrm{rm}$-level heterogeneities from raw price data by retrieving the residuals from a price regression involving \rm-level \xed effects. ${ }^{9}$ Wildenbeest (2011) veri囚es the theoretical validity of the procedure in a setting of unit demand and zero advertising costs where the $\nabla r m s$ differ in quality and costs, but share the same value-cost margin. However, our more general framework shows how the procedure is invalid (i) for downward-sloping demand (because the relationship between $\bigotimes$ rms $\$ offered prices and utilities becomes nonlinear), and (ii) under unit demand outside Wildenbeest $\$$ condition (because the $\nabla r m s$ no longer offer the same average utility). Moreover, we then offer the basis for modi冈ed methodologies that may be applied instead.

SectionХIIB uses the framework to study a family of games where each $\nabla$ rm® share of nonshoppers is determined endogenously as a function of the $\bigotimes$ rms $\$ actions prior to sales competition. Among other examples, such actions are consistent with forms of persuasive advertising, sales force methods, and obfuscation. Starting with Chioveanu (2008), Carlin (2009), and Wilson (2010), some related streams of

\footnotetext{
${ }^{8}$ Existing empirical studies often focus on different factors affecting rms $\bigotimes$ use of sales, such as market information, competition, or rivals $\bigotimes$ behavior (e.g., Shankar and邓Bolton 2004, Ellickson and邓Misra 2008, Lewis 2008, and Chandra and区Tappata 2011).

${ }^{9}$ The residuals are then used in (i) reduced-form studies of price dispersion (e.g., Sorensen 2000; Brown and Goolsbee 2002; Lach 2002; Barron, Taylor, and Umbeck 2004; Lewis 2008; Chandra and Tappata 2011; Sherman and Weiss 2017; and Pennerstorfer et\al. 2020), or (ii) structural estimations (e.g., Wildenbeest 2011; Moraga-Gonz囚lez, S囚ndor, and囚Wildenbeest 2013; Allen, Clark, and Houde 2014; Giulietti, Waterson, and

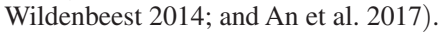


literature have become popular in recent years. ${ }^{10}$ However, almost all such models are based upon simple market settings with zero costs of informative advertising. An exception is Baye and Morgan (2009), but due to the consequent dif囚culties of analyzing asymmetries, they are unable to consider all possible subgames. Instead, they show the existence of a continuum of symmetric Nash equilibria and a unique symmetric equilibrium in secure strategies. In contrast, our framework can characterize the unique symmetric subgame perfect Nash equilibrium across a general market setting. Moreover, while their equilibria imply that an increase in the cost of informative advertising can have a positive, negative, or zero effect on the prior actions, our framework offers a unique empirically testable prediction. As the costs of informative advertising decrease, sales markets should experience (i) a reduction in loyalty-enhancing actions, such as persuasive advertising, and (ii) an increase in loyalty-reducing actions, such as some forms of obfuscation. This offers a $\bigotimes$ rst theoretical connection between the costs of informative advertising and equilibrium levels of persuasive advertising, and gives a new advertising-costs-based explanation for why rms increase their obfuscation tactics in response to advances in digital technology (e.g., Ellison and区Ellison 2009).

Finally, SectionХIIC provides some new comparative static results that could be utilized for future research regarding the effects of firm-level characteristics on sales and market performance. For instance, standard results show that an industry-wide increase in advertising costs deters the use of sales and raises $\bigotimes \mathrm{rms} \bigotimes$

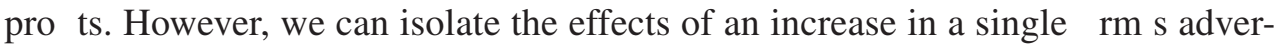
tising costs $\bigotimes$ we show that $\bigotimes$ rms still reduce their use of sales, but that it is rival rather than own advertising costs that matter in determining pro囚ts. Similarly, we isolate the effects of an increase in an individual $\nabla r m \bigotimes$ share of nonshoppers. In contrast to results under the traditional tiebreak rule (e.g., Arnold etखi 1. 2011), this induces the $\nabla r m$ to set lower average utility offers as more consistent with standard results under zero advertising costs (e.g., Narasimhan 1988). Lastly, we study

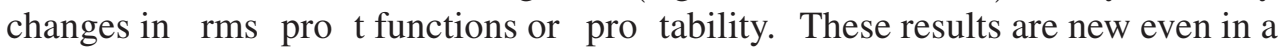
symmetric industry-wide setting $\bigotimes$ an industry-wide increase in pro囚tability, such as a reduction in costs or an increase in per-consumer demand, will always increase

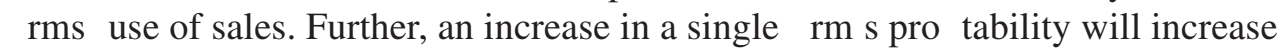
its sale probability and prompt it to use higher average offers in most common market settings.

Related Literature.-Armstrong and冈Vickers (2001) introduced competition in utility to study price discrimination in a symmetric, pure strategy setting. In contrast, we transfer their utility approach into an asymmetric (clearinghouse) model to study mixed strategy sales. Some past sales papers have referred to competition in utility (Simester 1997; Hosken and Reiffen 2007; Wildenbeest 2011; Dubovik and Janssen 2012; Anderson, Baik, and Larson 2015). However, they only use it to compute equilibria in speci $\ \mathrm{c}$ settings, and do not use the associated pro冈t function, $\pi(u)$, to explore any general results or implications. In a recent paper, Armstrong

\footnotetext{
${ }^{10}$ For a review, and for some wider related models, see Grubb (2015) and Spiegler (2016).
} 
and Vickers (2019) take a different line. They use a dual approach by writing an individual consumer $\varangle$ surplus as a function of the associated per-consumer pro $\bigotimes t$ to analyze the effects of price discrimination within a duopoly sales model with asymmetric shares of nonshoppers. Some other work also exists in nonclearinghouse settings. First, Anderson, Baik, and Larson (2015) allow for $\nabla$ rm heterogeneity in a model where $\bigotimes$ rms must advertise to earn positive pro\ts, and where all consumers

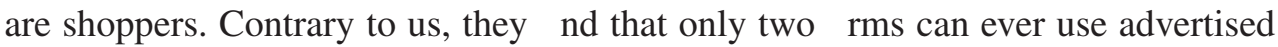
sales when $\nabla$ rms are heterogeneous. As such, they cannot analyze how market factors affect the number and type of rms that use sales, or connect to the larger theoretical or empirical clearinghouse literature. Instead, they focus on some interesting results regarding equilibrium selection and welfare. Second, some papers consider clearinghouse-style frameworks but under an assumption of horizontally differentiated products (e.g., Galeotti and囚Moraga-Gonz囚lez 2009, Moraga-Gonz囚lez and Wildenbeest 2012). These papers exhibit pure strategy price equilibria without price dispersion, and therefore do not share our focus on sales.

\section{Model}

Let there be $n \geq 2$ खrms, $i \in\{1, \ldots, n\}$. Also suppose there is a unit mass of risk-neutral consumers that have a zero outside option. Each $\ \mathrm{rm} i$ competes by choosing a utility offer (net of any associated payments), $u_{i} \in \mathbb{R}_{\geq 0}$. All consumers have identical preferences and so each consumer values $\bigotimes$ rm $i \bowtie$ offer at precisely $u_{i} \cdot 11$

The maximum possible proØt that $\bigotimes \mathrm{rm} i$ can extract per consumer when providing an offer, $u_{i}$, is de囚ned as $\pi_{i}\left(u_{i}\right)$. Following Armstrong and Vickers (2001), the exact source of utility and form of pro囚t function can depend upon a rich set of demand, product, and cost conditions. ${ }^{12}$ We assume that $\pi_{i}\left(u_{i}\right)$ is independent of the number of consumers served. ${ }^{13}$ Further, to ensure that any sales equilibrium is well-behaved, we make some mild technical assumptions: (i) $\pi_{i}\left(u_{i}\right)$ is strictly quasi-concave

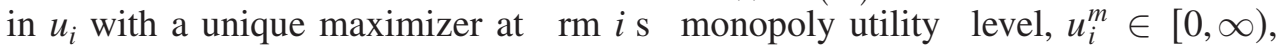
(ii) $\pi_{i}\left(u_{i}^{m}\right) \equiv \pi_{i}^{m}>0$, (iii) $\pi_{i}\left(u_{i}\right)$ is twice continuously differentiable for all $u_{i}>u_{i}^{m}$, and (iv) there exists a \nite break-even utility $>u_{i}^{m}$, where $\pi_{i}\left(\right.$ 洒 $\left._{i}\right)=0$.

Consumers are initially uninformed about $\bigotimes$ rms $\bigotimes$ utility offers. However, each 冈rm can choose whether or not to advertise in order to inform consumers of its offer, $\eta_{i} \in\{0,1\}$. In line with some previous versions of the clearinghouse model

\footnotetext{
${ }^{11}$ This assumption of identical preferences is standard within the mixed strategy sales literature. If, in contrast, consumers $\bigotimes$ preferences were suf $\bigotimes$ ciently heterogeneous, then any mixed strategy sales equilibrium would be replaced by a pure strategy nonsales equilibrium.

${ }^{12}$ As two simple examples, consider the following, where $\operatorname{\nabla rm} i$ sells a single good at price $p_{i}$ with marginal cost $c_{i}$. First, suppose each consumer has a unit demand and values $\nabla \mathrm{rm} i \mathbb{\varangle}$ good at $V_{i}$. Firm $i \mathbb{\varangle}$ utility offer is the associated consumer surplus, $u_{i}=V_{i}-p_{i}$, while its pro囚ts per consumer equal $\pi_{i}\left(u_{i}\right)=V_{i}-c_{i}-u_{i}$. Second, let each con-

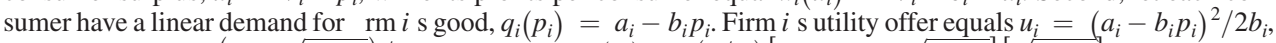
and by using $p_{i}=\left(a_{i}-\sqrt{2 b_{i} u_{i}}\right) / b_{i}$, one can write $\pi_{i}\left(u_{i}\right)=\left(1 / b_{i}\right)\left[a_{i}-b_{i} c_{i}-\sqrt{2 b_{i} u_{i}}\right]\left[\sqrt{2 b_{i} u_{i}}\right]$. Further examples including more general downward-sloping demand, multiproduct $\bigotimes$ rms, two-part tariffs, and nonprice sales are detailed in Appendix A.

${ }^{13}$ Similar to Armstrong and Vickers (2001), this is needed for the pro囚t function to remain well-de区ned. However, it rules out some empirically relevant features such as scale economies or capacity constraints.
} 
(e.g., Baye, Morgan, and Scholten 2004a), we assume (i) all advertising must be truthful, (ii) any advert is observed by all relevant consumers, and (iii) \rms $\nabla$ advertising costs are exogenous. However, in contrast to the previous literature, we also assume that (iv) advertising costs can differ across $\bigotimes_{\mathrm{rm} s}$, as consistent with different

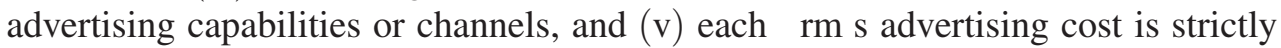
positive, $A_{i}>0 \forall i .^{14}$

There are two types of consumers, nnonshoppers $\bigotimes$ and $\bigotimes$ shoppers,, in respective proportions, $\theta \in(0,1)$ and $(1-\theta)$. Nonshoppers ignore all adverts. They simply visit their designated $\bigotimes$ local $\nabla$ rm and buy according to their underlying demand function, or exit. Our framework allows the $\mathrm{rrms}_{\mathrm{r}}$ to have asymmetric shares of nonshoppers, $\theta_{i}>0$, with $\sum_{i=1}^{n} \theta_{i}=\theta$. In contrast, the remaining 凶shopper $\otimes$ consumers pay attention to adverts and can buy from any $\nabla \mathrm{rm}$. However, to simplify exposition, we assume that shoppers can only visit one $\nabla r m$. Hence, shoppers choose between (i) visiting an advertising 冈rm to buy from its known utility offer, (ii) visiting a nonadvertising 冈rm to discover its utility offer and potentially buy, or (iii) exiting the market immediately. ${ }^{15}$

We analyze the following game. In stage 1, each $\nabla r m$ chooses its utility offer, $u_{i} \in \mathbb{R}_{\geq 0}$, and its advertising decision, $\eta_{i} \in\{0,1\}$. To allow for mixed strat-

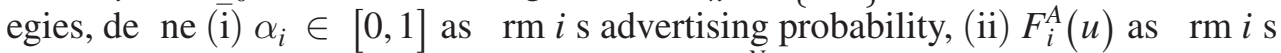

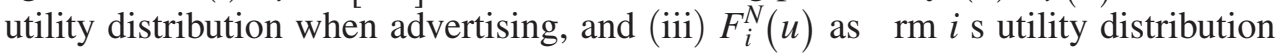
when not advertising, both on support $\mathbb{R}_{\geq 0}$. In stage 2 , consumers observe any adverts, form beliefs about the (expected) utility provided by any nonadvertising 冈rm, $u_{i}^{e}$, and then make their visit and purchase decisions in accordance with the strategies outlined above.

We de囚ne a $\bigotimes$ tie $\bigotimes$ as any situation where the shoppers are indifferent over visiting a set of $\nabla_{\mathrm{rms}}, T$, where $|T| \in[2, n]$. Any $\nabla_{\mathrm{rm}}$ within the tied set, $i \in T$, must have advertised (or be expected to offer) a common level of utility, $u$, while any 冈rm outside the tie, $j \neq T$, must have advertised (or be expected to offer) a utility strictly lower than $u$. A $\otimes t i e b r e a k$ rule $\otimes$ then assigns the probability (or proportion) with which the shoppers visit each tied $\bigotimes \mathrm{rm}$. In particular, for any $i \in T$, let $\bigotimes \mathrm{rm} i \mathbb{\varangle}$ \tiebreak probability, $\bigotimes x_{i}(\eta, u, T) \in[0,1]$, depend upon the tied $\nabla$ rms $\bigotimes$ advertising decisions, $\eta=\left\{\eta_{i}\right\}_{i \in T}$, the tied utility level, $u$, and the set of tied $\otimes \operatorname{rms}, T$, where $\sum_{i \in T} x_{i}(\eta, u, T)=1$.

The existing literature assumes that shoppers (i) trade exclusively and symmetrically with advertised $\nabla \mathrm{rm}(\mathrm{s})$ in any tie between advertised and nonadvertised 冈rms, and (ii) trade symmetrically with all tied \rms in any other form of tie. This can be summarized as follows for $n=2$ : $x_{1}((1,0), u,\{1,2\})=1, x_{1}((0,1), u$, $\{1,2\})=0$, and $x_{1}((1,1), u,\{1,2\})=x_{1}((0,0), u,\{1,2\})=0.5$ for all $u$. In contrast, while we also assume that $x_{i}(\cdot)$ is independent of $u$, we depart from the literature区 approach in two ways.

\footnotetext{
${ }^{14}$ Strictly positive advertising costs help ensure that each $\bigotimes \mathrm{rm}$ refrains from advertising with positive probability in our later equilibrium. This is needed for our tiebreak rule to be effective in providing tractability. See footnote 22 for more.

${ }^{15}$ These assumptions can be substantially generalized by allowing shoppers to visit rms sequentially pro-

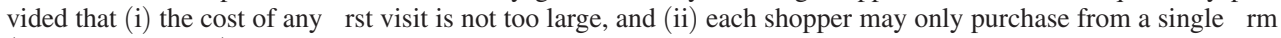
(one-stop shopping). For technical details see online Appendix C2.
} 
First, we assume that the tiebreak probabilities are independent of $\nabla r m s \rrbracket a d v e r t i s-$ ing decisions. In particular, Assumption X lets the tiebreak probabilities depend only on the set of tied $\bigotimes$ rms, $T$. While this assumption may be restrictive in some situations, it permits suf囚cient \exibility for us to manipulate the tiebreak rule. Furthermore, Assumption X remains consistent with our context where shoppers receive all adverts before making their visit decisions and so have no reason to favor advertising $\nabla \mathrm{rms}:{ }^{16}$
(Assumption X)
$x_{i}(\eta, u, T)=x_{i}(T) \quad \forall \eta, u$.

Second, instead of assuming that the tiebreak probabilities are otherwise symmetric, $x_{i}(T)=|T|^{-1}$, we build on the concept of endogenous sharing rules (Simon and Zame 1990) to specify them endogenously as part of the game equilibrium. Hence, we focus on perfect Bayesian equilibria (PBE), where in addition to specifying the players囚 equilibrium strategies and beliefs, we also specify the pro囚le of equilibrium tiebreak probabilities, $x^{*}(T)=\left\{x_{1}^{*}(T), \ldots, x_{n}^{*}(T)\right\}$, for all possible $T$. As detailed below, this approach, together with Assumption X, will allow us to manipulate the tiebreak probabilities to help improve equilibrium tractability.

Finally, we discuss two remaining assumptions. First, while our framework offers a signi囚cant increase in generality, it cannot avoid an assumption that is implicit across the entire previous literature. We are the 『rst to state it:
(Assumption U)
$u_{i}^{m}=u^{m} \quad \forall i$.

This does not require $\bigotimes$ rms to have the same monopoly price or the same monopoly proØts, only the same level of monopoly utility. However, Assumption U is not innocuous. Although it is trivially satis冈ed under unit demand or two-part tariffs because $u_{i}^{m}$ is then always zero (given our assumption that all consumers have identical preferences), it is restrictive under downward-sloping demand and linear prices. Here, one must either (i) restrict attention to symmetric pro®t functions $\pi_{i}(u)=\pi(u)$, or (ii) introduce some binding lower bound on $\triangle \mathrm{rms} \bigotimes$ utility offers, $u^{\min } \geq \min \left\{u_{i}^{m}, \ldots, u_{n}^{m}\right\}$, as consistent with an unmodeled competitive

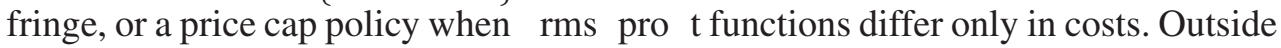
Assumption $\mathrm{U}$, the power of our tiebreak approach is lost. ${ }^{17}$

Second, we let all $\nabla r m s$ have some basic potential to use advertised sales. Speci凶cally, we let each $₫ \mathrm{rm} i \bowtie$ pro囚ts from not advertising with $u_{i}=u^{m}$ and selling only to its nonshoppers, $\theta_{i} \pi_{i}^{m}$, be less than its pro囚ts from advertising an offer just above $u^{m}$ and gaining the shoppers, $\left[\theta_{i}+(1-\theta)\right] \pi_{i}^{m}-A_{i}$. The resulting Assumption A is relatively mild and just ensures that each $₫ \mathrm{rm} \llbracket$ advertising cost is not prohibitively large:
(Assumption A)
$A_{i} \leq(1-\theta) \pi_{i}^{m} \quad \forall i$

\footnotetext{
${ }^{16}$ Footnote 19 also later explains how Assumption X can be partially relaxed.

${ }^{17}$ Suppose $u_{i}^{m}>u^{m}$ for some $i$. In the event where all $\mathrm{rms}$ set their monopoly utility, the shoppers will then strictly prefer to visit $\bigotimes_{r m} i$. As later explained, this implies there will be no ties in equilibrium. Thus, the tiebreak probabilities are redundant and cannot be used to ensure a tractable equilibrium.
} 


\section{Equilibrium Analysis}

SectionXIIA considers some preliminary $\bigotimes$ ndings before SectionХIIB provides some results on the equilibrium tiebreak probabilities. Section囚IC then completes the equilibrium analysis for duopoly $(n=2)$ before Section囚ID tackles the more complex case of a broader oligopoly $(n>2)$. Any formal proofs are listed in online Appendix B.

\section{A. Preliminary Results}

LEMMA 1: In any equilibrium, each firm $i$ must set $u_{i}=u^{m}$ if it does not advertise, $\eta_{i}=0$, and set $u_{i}>u^{m}$ if it advertises, $\eta_{i}=1$.

Any 『rm that does not advertise cannot use its unobserved utility offer to attract more consumers. Instead, it will $\bigotimes$ nd it optimal to set the monopoly utility level, $u^{m}$, because its nonshoppers and any visiting shoppers cannot visit elsewhere. In addi-

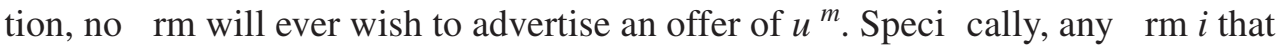
advertises $u^{m}$ (with positive probability) could pro囚tably deviate by not advertising. By doing so, it would reduce its advertising costs, $A_{i}>0$, while having no impact on its units sold since $x_{i}(T)$ is independent of advertising decisions via Assumption X.

Lemma 1 offers several implications. First, in equilibrium, if no advert is observed from $\bigotimes \mathrm{rm} i$, then shoppers must correctly believe $u_{i}^{e}=u^{m}$. Moreover, if no adverts are observed from any $\nabla \mathrm{rm}$, then shoppers must believe that all the $\nabla \mathrm{rms}$ are tied with $u_{i}^{e}=u^{m}$ for all $i$ via Assumption U. Second, if $\triangle \mathrm{rm} i$ advertises, $\bigotimes \mathrm{rm} i \otimes$ lowest advertised utility will always be strictly larger than its nonadvertised utility, $u^{m}$.

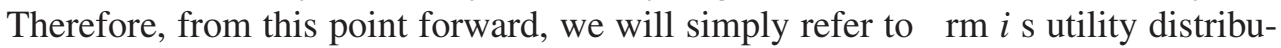
tion unconditional on advertising, $F_{i}(u)$, where $\nabla \operatorname{rm} i$ sets $u^{m}$ without advertising with probability $1-\alpha_{i}=F_{i}\left(u^{m}\right) \in[0,1]$, and uses advertised sales on $u>u^{m}$ with total probability $\alpha_{i}$.

Firm $i$ will then be said to use 囚sales $\bigotimes$ if it advertises an offer above $u^{m}$ with positive probability, $\alpha_{i}>0$. In any given equilibrium, we will refer to $k^{*}$ as the number of $\nabla$ rms that use sales and $K^{*}$ as the set of $\nabla r m s$ that use sales. A $\bigotimes$ sales equilibrium $\bigotimes$ will be de冈ned as any equilibrium where $k^{*} \geq 1$. In any given sales equilibrium, we will denote $\stackrel{\mathbb{U}}{\mathrm{X}}>u^{m}$ as the minimum level of $u$ for which $F_{i}(u)=1$ for all $i$. By adapting standard arguments, we can state the following result.

LEMMA 2: In any sales equilibrium, at least two firms use sales, $k^{*} \geq 2$, and for at least two firms $i$ and $j, u$ is a point of increase of $F_{i}(u)$ and $F_{j}(u)$ at any $u \in\left(u^{m}, \bar{u}\right]$. Any firm which uses sales, $i \in K^{*}$, has no point masses in $F_{i}(u)$ for $u>u^{m}$ and advertises with an interior probability, $\alpha_{i}=1-F_{i}\left(u^{m}\right) \in$ $(0,1)$. When $n=2$, any sales equilibrium has both firms advertising on $\left(u^{m}, \bar{u}\right]$ without gaps.

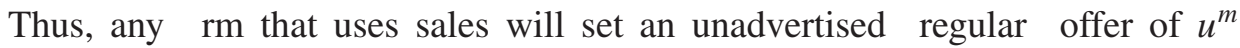
with probability $\left(1-\alpha_{i}\right) \in(0,1)$, together with randomized discounted offers 
$u \in\left(u^{m}, \mathbb{l}_{0}\right.$ with probability $\alpha_{i} \cdot{ }^{18}$ When $n=2$, Lemma 2 demonstrates that any sales equilibrium will involve both $\nabla$ rms using sales on the same full support $\left(u^{m}\right.$, 即. However, when $n>2$, similar to the insights of Baye, Kovenock, and de Vries (1992) for zero advertising costs, it implies that there may be multiple forms of sales equilibria with $\triangle \mathrm{rms}$ using different supports. Indeed, provided at least two \rms mix on any given interval within $\left(u^{m}, \mathbb{\|}\right]$, other advertising $\nabla$ rms need not be active on the same interval. Hence, to avoid these signi冈cant complications and potential multiplicities when $n>2$, we follow Chioveanu (2008) by focusing only on sales equilibria where all advertising $\bigotimes$ rms use the full convex support, $\left(u^{m}, \bigotimes_{l}\right]$. Hence, for all $i$ with $\alpha_{i}>0$, we assume $u$ is a point of increase of $F_{i}(u)$ for all $u \in$ $\left(u^{m}, \prod_{1}\right.$.

\section{B. Equilibrium Tiebreak Probabilities}

LEMMA 3: Ties can only occur with positive probability in equilibrium when all firms refrain from advertising, $\eta_{i}=0 \forall i$.

In contrast to the existing literature where ties are possible between nonadvertising and advertising Ørms, Lemma 3 shows that ties are only possible in our model when all rrms choose not to advertise. Hence, the only tiebreak probability that can be relevant in equilibrium is $x_{i}(N)$ where $N$ denotes the set of all $\nabla \mathrm{rms}$, and so from this point forward, we simply denote $x_{i}(N) \equiv x_{i}$, and $x^{*}=\left\{x_{1}^{*}, \ldots, x_{n}^{*}\right\}$ as a set of equilibrium tiebreak probabilities. This difference to the literature arises from our Assumption X, which ensures that the tiebreak rule is independent of $\nabla \mathrm{rms} \bigotimes$ advertising decisions. 19

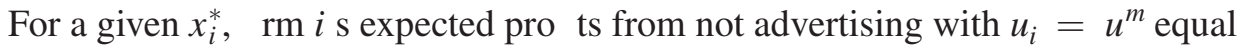

$$
\pi_{i}^{m}\left[\theta_{i}+(1-\theta) x_{i}^{*} \prod_{j \neq i}\left(1-\alpha_{j}\right)\right] .
$$

Firm $i$ will always trade with its $\theta_{i}$ nonshoppers, but it will also trade with the $(1-\theta)$ shoppers if (i) all other $\bigotimes$ rms also choose not to advertise, which occurs with probability $\Pi_{j \neq i}\left(1-\alpha_{j}\right)$, and (ii) the shoppers visit $i$ in the subsequent tie, which occurs with tiebreak probability $x_{i}^{*}$.

If $₫ \mathrm{rm} i$ uses sales in equilibrium, then we know from Lemma 2 that it must use an interior probability, $\alpha_{i} \in(0,1)$. Hence, under the requirements of a mixed strategy equilibrium and our assumption that all advertising 囚rms use the full support $\left(u^{m}, \bigotimes_{i}\right]$, \rm $i$ must expect to earn the same level of equilibrium pro®ts, $\AA_{i}$, from

\footnotetext{
${ }^{18}$ This sales behavior is consistent with the empirical evidence cited in the introduction. While Hitsch, Hortacsu,

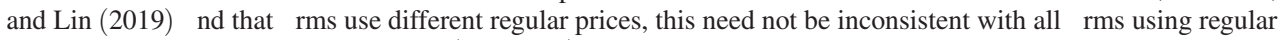
offers of $u^{m}$ provided the $\triangle \mathrm{rms}$ differ in (perceived) quality or service levels.

${ }^{19}$ Assumption X can be partially relaxed. If, instead, advertising 冈rms were assigned (slightly) lower tiebreak probabilities than nonadvertising $\triangle \mathrm{rms}$, then our results would not change区 advertising $u^{m}$ would still be domi-

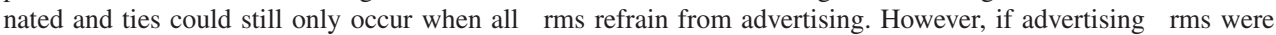
assigned signi $\bowtie$ cantly higher tiebreak probabilities than nonadvertising 冈rms, we would move closer to the existing literature. Speci囚cally, advertising $u^{m}$ would not be dominated and so ties could also exist between advertising and nonadvertising $\bigotimes$ rms at $u^{m}$ in ways that would generate the literature associated loss in equilibrium tractability.
} 
(i)凹setting $u_{i}=u^{m}$ and not advertising, and (ii) advertising any $u_{i} \in\left(u^{m}\right.$, 樼. We can then state the following.

LEMMA 4: Consider any sales equilibrium with a given set of tiebreak probabilities, $x^{*}$. Then, if firm i uses sales, its equilibrium profits are uniquely defined as

$$
\bigcap_{i}=\theta_{i} \pi_{i}^{m}+\frac{x_{i}^{*}}{1-x_{i}^{*}} A_{i} \text {. }
$$

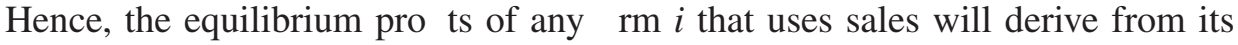
share of nonshoppers, $\theta_{i}$, its advertising costs, $A_{i}$, and its equilibrium tiebreak probability, $x_{i}^{*}$. To begin to understand more about the equilibrium tiebreak probabilities, it is useful to now de冈ne the following expressions, where $\theta_{-i}=\theta-\theta_{i}$ refers to the total share of nonshoppers that are not designated to $\operatorname{\nabla rm} i$ :

$$
\begin{aligned}
\chi_{i}(u) & \equiv 1-\frac{A_{i}}{\pi_{i}(u)\left(1-\theta_{-i}\right)-\theta_{i} \pi_{i}^{m}}, \\
\tilde{u}_{i} & \equiv \pi_{i}^{-1}\left(\frac{\theta_{i} \pi_{i}^{m}+A_{i}}{1-\theta_{-i}}\right) .
\end{aligned}
$$

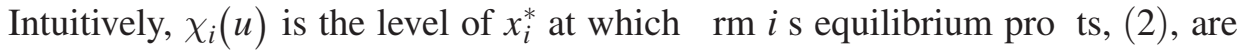
equal to the maximum pro®ts that $\bigotimes \mathrm{rm} i$ could obtain from advertising an offer, $u$, and successfully attracting all the shoppers, $\pi_{i}(u)\left(1-\theta_{-i}\right)-A_{i}$. Further, $\tilde{u}_{i}$ can then be understood as the level of utility at which $\chi_{i}(u)=0$, where $\nabla \operatorname{rm} i \mathbb{\text { max}}$ imum pro®ts from advertising are equal to its lowest possible pro®ts from not advertising, $\theta_{i} \pi_{i}^{m}>0$. Hence, $\nabla \operatorname{rm} i$ will never advertise $u_{i}>\tilde{u}_{i}$. Formally, we de冈ne $\chi_{i}(u)$ on $\left[u^{m}, \tilde{u}_{i}\right]$ with $\chi_{i}^{\prime}(u)<0$. Using Assumption $\mathrm{A}$, we then know that $\chi_{i}\left(u^{m}\right)=1-\left(A_{i} /\left((1-\theta) \pi_{i}^{m}\right)\right) \in[0,1)$ is weakly larger than $\chi_{i}\left(\tilde{u}_{i}\right)=0$, or equivalently, $\tilde{u}_{i} \geq u^{m}$ for all $i$.

LEMMA 5: Consider any sales equilibrium with a given upper utility bound 冈 $>u^{m}$. Then, if firm $i$ uses sales, $(i)$ the upper bound must satisfy $\| \leq \tilde{u}_{i}$, and (ii) firm i's equilibrium tiebreak probability is uniquely defined as

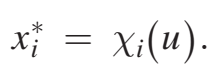

If $\nabla$ rm $i$ uses sales on $u_{i} \in\left(u^{m}, \rrbracket\right]$, then we know from above that $\tilde{u}_{i}$ must

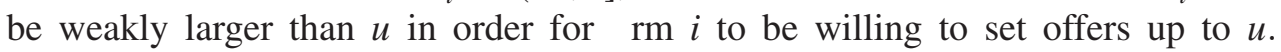
Moreover, if $\bigotimes \mathrm{rm} i$ uses sales, then its equilibrium pro®ts, $\prod_{i}$, must equal its expected pro®ts from advertising any $u_{i} \in\left(u^{m}, \prod^{\boxplus}\right.$. Hence, by setting $\prod_{i}$ equal to its expected pro囚ts from advertising $₫$, where it would attract the shoppers for sure, $\left(1-\theta_{-i}\right) \pi_{i}(\mathbb{\sharp})-A_{i}$, we know that $\nabla \mathrm{rm}$ i equilibrium tiebreak probability, $x_{i}^{*}$, must equal $\chi_{i}(\mathbb{\pi})$; any other $x_{i} \neq \chi_{i}(\mathbb{\sharp})$ is incompatible with a sales equilibrium under our assumptions. Thus, the equilibrium tiebreak probabilities for any $\nabla r m s$ using sales must ensure that all such $\bigotimes$ rms have exactly the same incentive to employ the common upper utility bound, $\mathrm{\bigotimes}$. 
To help understand this further, Drst consider a fully symmetric setting. Here, the \rms already have identical incentives and so (5) implies that any rms that engage in sales will share a common equilibrium tiebreak probability. More importantly, now consider an example asymmetric setting where only $\nabla$ rms 1 and 2 engage in sales, and where $\nabla \mathrm{rm} 1$ is relatively more willing to advertise higher utilities, $\tilde{u}_{1}>\tilde{u}_{2} \cdot{ }^{20}$ In equilibrium, to ensure that the $\nabla$ rms have the same incentive to adopt a common upper bound, (5) implies that $\nabla_{r m} 1$ must be assigned a larger equilibrium tiebreak

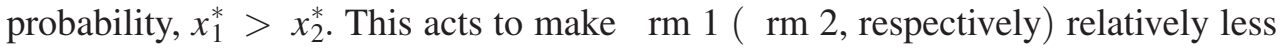
(more, resp.) aggressive by enhancing (reducing, resp.) its expected payoffs from not advertising. However, as later shown, it is not the case that $x_{1}^{*}$ and $x_{2}^{*}$ prompt the \rms to play symmetric strategies. Thus, the equilibrium tiebreak probabilities do not fully neutralize the $\bigotimes$ rms $\bigotimes$ heterogeneities, they just partially offset them. ${ }^{21}$

$$
\text { C. Duopoly }(n=2)
$$

As now formalized, the equilibrium under duopoly is unique and takes one of two forms. When advertising costs are suf冈ciently low, there is a sales equilibrium where both 冈rms engage in sales, otherwise, there is a nonsales equilibrium.

LEMMA 6: When $n=2$, any sales equilibrium has a unique upper utility bound, 风, (implicitly) defined by (6), and each firm's advertising probability and offer distribution are uniquely defined by (7) and (8):

$$
\begin{aligned}
\chi_{1}(\text { 冈 })+\chi_{2}(\bowtie) & =1, \\
\alpha_{i} & =1-\frac{A_{j}}{x_{i}^{*}(1-\theta) \pi_{j}^{m}}, \\
F_{i}(u) & =\frac{\theta_{j}\left(\pi_{j}^{m}-\pi_{j}(u)\right)+\left(A_{j} / x_{i}^{*}\right)}{(1-\theta) \pi_{j}(u)} .
\end{aligned}
$$

When $n=2$, previous results have shown that any sales equilibrium must involve both $\nabla \mathrm{rms}$ and that $x_{i}^{*}=\chi_{i}(\sharp)$. Hence, as the tiebreak probabilities must sum to one, the unique equilibrium upper bound must satisfy (6). One can then derive each

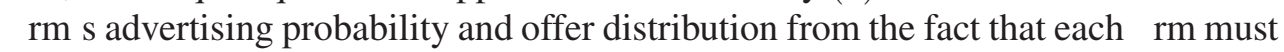

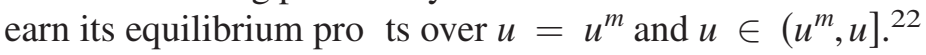

\footnotetext{
${ }^{20}$ From inspection of (4), this could arise because $\otimes \mathrm{rm} 1$ has a relatively lower advertising cost, $A_{1}$, a relatively lower share of nonshoppers, $\theta_{1}$, a relatively lower level of per-consumer pro®ts at $u^{m}, \pi_{1}^{m}$, and/or a relatively higher level of per-consumer pro®ts at $\geqq, \pi_{1}(\rrbracket)$.

${ }^{21}$ One may ask why the shoppers should behave in accordance with (5). Similar questions arise within the wider concept of endogenous sharing rules. As noted by Simon and Zame (1990, 863), QThe answer is, as always, that equilibrium theory never explains why any agents would act in any particular way. Equilibrium theory is intended to explain how agents behave, not why $\boldsymbol{Q}$

${ }^{22}$ In an extreme case where the $\nabla \mathrm{rms}$ are asymmetric but $A_{i}=A_{j} \rightarrow 0$, the only way for the $\nabla \mathrm{rms}$ to share a common upper utility bound is for $x_{i}^{*} \rightarrow 1$ and $x_{j}^{*} \rightarrow 0$. This limit equilibrium converges to the equilibrium of a
} 
Proposition 1 now shows that the characterized sales equilibrium exists uniquely if advertising costs are suf冈ciently low, $\left(A_{1} / \pi_{1}^{m}\right)+\left(A_{2} / \pi_{2}^{m}\right)<1-\theta$. In contrast, if advertising costs are higher, the rrms are deterred from competing against each other $\bigotimes$ instead, there exists a unique nonsales equilibrium where both 冈rms select $u^{m}$ and refrain from advertising.

PROPOSITION 1: Given Assumptions X, $U$, and $A$, the game has the following unique equilibrium (where consumers always expect nonadvertising firms to offer $\left.u^{m}\right)$ :

(i) If advertising costs are low, $\left(A_{1} / \pi_{1}^{m}\right)+\left(A_{2} / \pi_{2}^{m}\right)<1-\theta$, each firm $i$ offers $u_{i}=u^{m}$ and does not advertise with probability $\left(1-\alpha_{i}\right) \in(0,1)$ according to (7), and advertises a sale offer $u_{i} \in\left(u^{m}, \bar{u}\right]$ according to (8) with probability $\alpha_{i}$, where the upper bound, $\bar{u}$, solves $(6)$, and firm $i$ 's equilibrium tiebreak probability, $x_{i}^{*}=1-x_{j}^{*} \in(0,1)$, is given by $(5)$.

(ii) If advertising costs are high, $\left(A_{1} / \pi_{1}^{m}\right)+\left(A_{2} / \pi_{2}^{m}\right) \geq 1-\theta$, both firms offer $u_{i}=u^{m}$ and never advertise, $\alpha_{i}=0$, and firm i's equilibrium tiebreak probability equals $x_{i}^{*}=1-x_{j}^{*} \in\left[\chi_{i}\left(u^{m}\right), 1-\chi_{j}\left(u^{m}\right)\right]$.

One can demonstrate the implications of Proposition 1 by specifying the exact source of utility and pro®t function. As a very simple example, consider a symmetric setting with unit demand. Following footnote 12 , let $u_{i}=V-p_{i}$ and $\pi\left(u_{i}\right)=V-c-u_{i}$, where $u^{m}=0$ and $\pi^{m}=V-c$. Proposition 1 then implies a clearinghouse equilibrium with $x_{i}^{*}=0.5, \AA_{i}=(\theta(V-c) / 2)+A$,

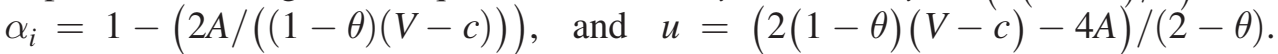
By using $F_{i}(p)=1-F_{i}(u)$, one can then further derive $F_{i}(p)=1-$ $(\theta(V-p)+4 A) /(2(1-\theta)(p-c))$, with $p^{m}=V-u^{m}=V$ and $\underline{p}=V-\not 囚$ $=c+(\theta(V-c)+4 A) /(2-\theta)$. Further details and additional example settings involving downward-sloping demand, and multiproduct $\bigotimes$ rms are included in Appendix A.

Appendix A also shows how Proposition 1 can be used to characterize two common forms of sales that have remained unstudied within the clearinghouse literature. First, it characterizes sales behavior when $\nabla r m s$ use two-part tariffs. Existing theoretical work is very limited on this $\bigotimes$ we only know of Hendel, Lizzeri, and Roketskiy (2014), which shows how sales with nonlinear prices can emerge in a dynamic context with storable goods. In contrast, our framework considers a simpler clearinghouse setting while allowing for full asymmetry. We show that equilibrium sales will involve marginal cost pricing and rms mixing between not advertising a high $\nabla x e d$ fee and advertising a stochastic lower $\nabla x e d$ fee. While there is little empirical analysis available, our predictions are consistent with several anecdotal examples and some wider forms of evidence. ${ }^{23}$

\footnotetext{
model that allows for $A=0$ explicitly without our tiebreak rule. See online Appendix C1 for full details.

${ }^{23}$ In practice, the use of such tariffs may also be driven by heterogeneous consumer preferences. However, consistent with our predictions, most UK suppliers of broadband, landline, and TV packages, as well as many gym
} 
Second, it characterizes sales when 冈rms hold prices constant but compete with some nonprice variable. This setting covers a broad set of commonly observed marketing practices, including (i) temporary extensions to package size or quantity, such as $₫ X \%$ Free $\bigotimes$ offers and $\bigotimes$ bonus packs, (ii) temporary increases in product quality or content, such as the inclusion of free items or Øpremiums, $\bigotimes$ and (iii) other temporary increases in product value, such as the use of consumer nnance deals, prize draws, or charity donations (see the discussions in Palazon and Delgado-Ballester 2009, Chen et $\$ 1$. 2012). As consistent with these phenomena, we show that equilibrium sales will

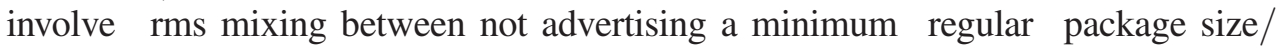
product value and advertising a sale with an increased package size/product value.

\section{D. $n>2$ Firms}

We now extend the analysis beyond the simpler case of duopoly to offer new results about the number and type of Ørms that use sales in markets with $n>2$ ⿶rms. Here, the sales literature with heterogeneous $\unrhd$ rms is scant because existing models quickly become intractable. Most notably, as part of their analysis, Baye, Kovenock, and de Vries (1992, Lemmas 7凶14') establish that only two \rms can ever engage in sales behavior in a unit-demand clearinghouse model with zero advertising costs when rrms differ in their shares of nonshoppers. The remaining \rms with relatively larger shares of nonshoppers are less willing to compete and prefer to always set high (nonsale) prices to their nonshoppers. This $₫$ nding has been extended to allow $\bigotimes$ rms to vary in their product values (Kocas and Kiyak 2006) or costs (Shelegia 2012).

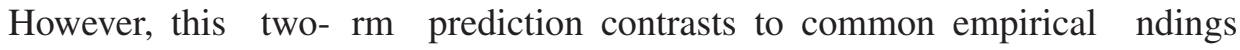
where multiple heterogeneous sellers exhibit sales behavior (e.g., Lach 2002, Lewis 2008, Chandra and Tappata 2011). Instead, within our more general framework, we now demonstrate how any number of heterogeneous $\bigotimes$ rms, $k^{*} \in[2, n]$, can engage in equilibrium sales. In particular, we explain the factors that determine the number of $\nabla r m s$ that use sales in equilibrium, and provide a broad characterization of the types of $\nabla$ rms that are likely to use sales, depending on their advertising costs, nonshopper shares, and pro囚t functions.

To proceed, we use (4) to index the $\nabla r m s$ in (weakly) decreasing order of $\tilde{u}_{i}$ from 1 to $n$, such that $\nabla \mathrm{rm} n$ is the least willing to advertise high utilities. We then focus on characterizing sales equilibria in two settings: (i) a quasi-symmetric setting where $u^{m}<\tilde{u}_{i}=\tilde{u}$ for all $i$, and (ii) a strict asymmetric setting where $u^{m}<\tilde{u}_{n}<\cdots<\tilde{u}_{1} \cdot{ }^{24}$

Unlike the duopoly case, the tiebreak probabilities can now generate potential sources of sales equilibrium multiplicity. This can arise for two reasons. First, sales equilibria are now possible where at least one $\nabla r m$ does not advertise. Here, Lemma 5 is insuf®cient to pin down a unique equilibrium value of $x_{i}^{*}$ for $\nabla \mathrm{rms}$ that never

facilities and sports clubs, offer sales with reduced monthly fees but unchanged prices for charged services. Our predictions are also consistent with a \nding in Giulietti, Waterson, and Wildenbeest (2014), which suggests that \rms play mixed strategies with the implied $\nabla$ nal bill $\bigotimes$ for an average consumer in the British electricity market where suppliers often employ two-part tariffs.

${ }^{24} \mathrm{~A}$ third setting where a subset of $\nabla \mathrm{rms}$ have the same $\tilde{u}$ but where some remaining $\nabla \mathrm{rms}$ differ in $\tilde{u}$ can also be analyzed but is omitted for brevity due to its unnecessary complications. 
advertise, $\alpha_{i}=0$. Second, equilibrium multiplicity can also exist at knife-edge cases where $\tilde{u}_{i}=\bigotimes$ for some $\bigotimes$ rm $i$. Here, Lemma 5 implies that $x_{i}^{*}=0$ such that 冈rm $i$ is indifferent between using sales or not. To avoid both of these ambiguities which are largely uninteresting from an economic perspective, we focus on sales equilibria where (i) \rms that never advertise receive a zero equilibrium tiebreak probability, $x_{i}^{*}=0$ if $\alpha_{i}=0$, and (ii) advertising $\nabla$ rms receive a positive equilibrium tiebreak probability, $x_{i}^{*}>0$ if $\alpha_{i}>0$. Lemma 7 now provides a preliminary step, before Proposition 2 summarizes our main equilibrium result.

LEMMA 7: Consider any sales equilibrium that satisfies our restrictions with a given upper utility bound, 良 $>u^{m}$. Firm $i$ uses sales if and only if $\tilde{u}_{i}>\mathbb{X}$. Hence, (i) if $k^{*}=n$ then $\mathbb{\boxplus} \in\left(u^{m}, \tilde{u}_{n}\right)$, and (ii) if $k^{*} \in[2, n)$ then $\in\left[\tilde{u}_{k^{*}+1}, \tilde{u}_{k^{*}}\right)$ and $K^{*}=\left\{1, \ldots, k^{*}\right\}$.

The basic intuition is straightforward $\bigotimes$ $\bigotimes \mathrm{rm} i$ will only be willing to engage in sales within a given sales equilibrium if the upper bound, $\mathbb{x}$, is lower than the maximum utility that $\bigotimes$ rm $i$ could possibly wish to advertise, $\tilde{u}_{i}$, from (4). From this logic, Lemma 7 then goes on to make two immediate statements about the number and identity of $\bigotimes$ rms that will use sales for a given $囚$. If all $\bigotimes$ rms use sales, $k^{*}=n$, then it must be that the upper bound is suf囚ciently low such that $\nabla \mathrm{rm} n$ is willing to use

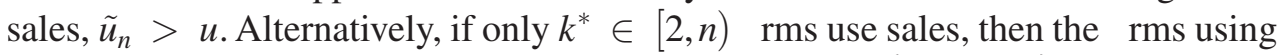
sales must be those with the highest values of $\tilde{u}_{i}, K^{*}=\left\{1, \ldots, k^{*}\right\}$. In particular, it must be that $\mathbb{\|} \in\left[\tilde{u}_{k^{*}+1}, \tilde{u}_{k^{*}}\right)$ such that $囚<\tilde{u}_{i}$ for $i \in K^{*}=\left\{1, \ldots, k^{*}\right\}$, but

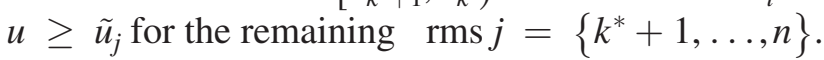

By using this together with an approach similar to Section邓IC, we now characterize a unique sales equilibrium under the assumption that a sales equilibrium exists. ${ }^{25}$ To avoid undue repetition of technical details, Proposition 2 jumps to the main result (see the proof for full details).

PROPOSITION 2: When a sales equilibrium exists under our restrictions, it is unique. In such an equilibrium, firms $i \leq k^{*}$ engage in sales with interior probabilities, $\alpha_{i} \in(0,1)$, while any remaining firms, $j>k^{*}$, never advertise $\alpha_{j}=0 ; k^{*}, x^{*}$, and $\mathrm{X}$ are uniquely defined as follows:

$$
\begin{aligned}
k^{*} & = \begin{cases}n & \text { if } \sum_{i=1}^{n} \chi_{i}\left(\tilde{u}_{n}\right)<1<\sum_{i=1}^{n} \chi_{i}\left(u^{m}\right) \\
k \in[2, n) & \text { if } \sum_{i=1}^{k} \chi_{i}\left(\tilde{u}_{k}\right)<1 \leq \sum_{i=1}^{k} \chi_{i}\left(\tilde{u}_{k+1}\right),\end{cases} \\
x_{i}^{*} & = \begin{cases}\chi_{i}(\mathbb{\sharp}) \in(0,1) & \text { if } i \leq k^{*} \\
0 & \text { if } i>k^{*},\end{cases} \\
\sum_{i=1}^{k^{*}} \chi_{i}(\mathbb{\sharp}) & =1 .
\end{aligned}
$$

\footnotetext{
${ }^{25}$ While Proposition 2 demonstrates equilibrium uniqueness, we are unable to prove existence for the general case when $n>2$ as it is dif囚cult to verify that $F_{i}^{\prime}(u)>0$ over the relevant $u$ for all $i \in K^{*}$. However, existence

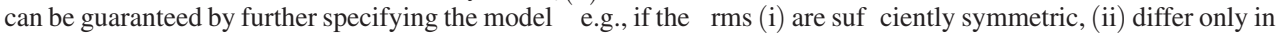
their advertising costs, or (iii) differ only in their pro®t functions when $\pi_{i}(u)=t_{i} \pi(u)$ where $t_{i}>0$. An explicit asymmetric example is provided later in the section.
} 
Before discussing the economic intuition and implications of Proposition 2, it is useful to provide a sketch of the technical proof. First, for a given $k^{*} \in[2, n]$, we derive the unique set of equilibrium tiebreaking probabilities, $x^{*}$ in (10), and the unique upper bound, 希 in (11). As the nonadvertising Vrms have $x_{i}^{*}=0$ by assumption, the values of $x_{i}^{*} \in(0,1)$ for the advertising 冈rms follow from Lemma 5 and must sum to one. Second, using Lemma 7, we then specify the conditions for the equilibrium upper bound, $₫$, to be consistent with the stated number of advertising 冈rms, $k^{*}$. In particular, for a given $k^{*}$, we require $(9)$ to ensure that $₫ \in\left(u^{m}, \tilde{u}_{n}\right)$ if $k^{*}=n$, and $\mathbb{\|} \in\left[\tilde{u}_{k^{*}+1}, \tilde{u}_{k^{*}}\right)$ if $k^{*} \in[2, n)$. Third, we show that (9) always speci冈es a unique equilibrium value of $k^{*} \in[2, n]$ provided advertising costs are suf $\mathbb{\Delta}^{-}$ ciently low: $\sum_{i=1}^{n} \chi_{i}\left(u^{m}\right)>1$ or equivalently, $\sum_{i=1}^{n}\left(A_{i} / \pi_{i}^{m}\right)<(n-1)(1-\theta) .{ }^{26}$ Finally, given $k^{*}$, the proof derives the unique advertising probabilities, $\alpha_{i} \in(0,1)$, utility distributions, $F_{i}(u)$, and pro囚ts, $\bar{\Pi}_{i}$.

To examine the economic intuition of Proposition 2, we now further discuss the number and type of rrms that use equilibrium sales, in turn.

The Number of Firms That Use Sales. $囚$ First, consider the quasi-symmetric case, where $\tilde{u}_{i}=\tilde{u}>u^{m}$ for all $i$. Here, from $(4), \chi_{i}(\tilde{u})=0$ for all $i$, and so the conditions in (9) can never be satis囚ed for $k^{*} \in[2, n)$. Instead, any sales equilibrium must have $k^{*}=n$. If two \rms wish to use sales, then they all wish to use sales. This equilibrium then resembles that under duopoly; all $\nabla r m s$ engage in sales on $\left(u^{m}, \mathbb{\prod}\right]$, and the unique set of equilibrium tiebreaking probabilities, $x^{*}$, ensures that each $\nabla r m$ has an identical incentive to adopt the common upper bound. However, the resulting tiebreak probabilities, utility distributions, and advertising probabilities need not be symmetric in equilibrium $\bigotimes$ they are only symmetric if the 冈rms also have identical advertising costs, nonshopper shares, and pro®t functions. Equilibrium existence can be further demonstrated with $F_{i}^{\prime}(u)>0 \forall i$ over the relevant range if the $\nabla r m s$ are suf囚ciently symmetric.

Now consider the strict asymmetric setting, where $u^{m}<\tilde{u}_{n}<\cdots<\tilde{u}_{1}$. Here, the intuition regarding $k^{*}$ is more complex. However, some broad insights can be gained by simplifying to symmetric advertising costs, $A_{i}=A \forall i$, where any changes in $A$ do not affect the ranking of $\nabla r m s$ in terms of $\tilde{u}_{i}$.

COROLLARY 1: Suppose firms are strictly asymmetric, $u^{m}<\tilde{u}_{n}<\cdots<\tilde{u}_{1}$, but advertising costs are symmetric, $A_{i}=A \forall i$. When a sales equilibrium exists, (i) only two firms use sales when advertising costs are sufficiently small, $k^{*}=2$ when $A \rightarrow 0$, but (ii) all firms use sales when advertising costs are moderate, $k^{*}=n$ when $A \rightarrow(n-1)(1-\theta) /\left(\sum_{i} 1 / \pi_{i}^{m}\right)$.

When $A \rightarrow 0$, our $\bigotimes$ ndings are in line with the existing literature $\$$ two- $\bigotimes$ rm result and generalize it to a broad range of market settings. When $A \rightarrow 0$, competition for the shoppers is Derce. Hence, the only way for any $\nabla r m s$ to have exactly the same incentives to employ a common upper bound is to give the $\nabla r m$ with the highest

\footnotetext{
${ }^{26}$ In contrast, if $\sum_{i=1}^{n} \chi_{i}\left(u^{m}\right) \leq 1$, then there is no solution for $k^{*} \in[2, n]$. Instead, in parallel to the duopoly case, there is a nonsales equilibrium with $k^{*}=0$ for appropriate values of $x^{*}$.
} 
incentive to advertise, $\nabla \mathrm{rm} 1$, almost all the shoppers in a tie, $x_{1}^{*} \rightarrow 1$. In equilibrium, only $\bigotimes \mathrm{rms} 1$ and 2 then use sales with $\mathbb{\|}=\tilde{u}_{2}$.

However, once we allow for higher advertising costs, the two- $\$ rm result becomes a special case of a new and more general relationship. Indeed, from (9), any number of heterogeneous $\nabla_{r m s} k^{*} \in[2, n]$ may now use sales in equilibrium. At the extreme, Corollary 1 states that all vrms can use sales provided advertising costs are moderate. This appears paradoxical at $\nabla$ rst because an increase in $A$ reduces the direct incentives for each $\bigotimes \mathrm{rm}$ to use sales, as evidenced by the associated reduction in $\tilde{u}_{i}$. However, the increase in $A$ also softens the competition for the shoppers and reduces $₫$ in a way that prompts $\bigotimes$ rms with lower $\tilde{u}_{i}$ to start using sales. Indeed, for moderate $A$, $囚$ can fall below $\tilde{u}_{n}$ such that all vrms use sales. Thus, if the costs of informative advertising fall in the digital era, then Corollary 1 suggests that fewer \rms might opt to use sales.

To conclude, we provide the following explicit example. Assume unit demand such that $\pi_{i}(u)=\Psi_{i}-u$ and $\pi_{i}^{m}=\Psi_{i}>0$, where $\Psi_{i}=V_{i}-c_{i}$ denotes the value-cost markup. Suppose $n=3$ and let the $\operatorname{\Delta rms}$ be symmetric aside from $\Psi_{1}>\Psi_{2}>\Psi_{3}>0$ such that $u^{m}<\tilde{u}_{3}<\tilde{u}_{2}<\tilde{u}_{1}$. The conditions in (9) then imply that $k^{*}=2$ if $A \leq \underline{A}$, and $k^{*}=3$ if $A \in(\underline{A}, \stackrel{A}{A}) .{ }^{27}$ Firm 1 has the largest equilibrium tiebreaking probability, but it still advertises with the highest probability and offers the highest average utility offers. Equilibrium existence can be demonstrated with $F_{i}^{\prime}(u)>0$ over the relevant range $\forall i \leq k^{*}$ provided the \rms are not too asymmetric. ${ }^{28}$

The Types of Firms That Use Sales.凶 To further explore the intuition of Proposition 2, we now consider its implications for the types of $\nabla r m s$ that are most likely to use sales. The existing literature only considers some speciðc dimensions under unit demand and zero advertising costs (e.g., Baye, Kovenock, and de Vries 1992; Kocas and Kiyak 2006; Shelegia 2012). However, in our general setting, we can offer a broad characterization. In particular, when $k^{*}<n$, Proposition 2 implies that the $\triangle \mathrm{rms}$ using sales will be the $\nabla \mathrm{rms}$ with the highest values of $\tilde{u}_{i}$ in (4). Corollary 2 then follows immediately and generally because $\tilde{u}_{i}$ is strictly decreasing in $A_{i}$ and $\theta_{i}$.

COROLLARY 2: Suppose a sales equilibrium exists with $k^{*}<n$. Ceteris paribus, the firms with the lowest advertising costs, $A_{i}$, and shares of nonshoppers, $\theta_{i}$, will use sales.

However, understanding how a $\nabla \mathrm{rm} \bigotimes$ pro®t function, $\pi_{i}(u)$, will impact its use

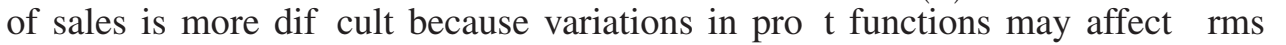
pro冈ts differently at different utility levels. To proceed, we focus on the following functional form, $\pi_{i}(u)=\pi\left(u, \rho_{i}\right)$, where $\pi(\cdot)$ is common across $\nabla \mathrm{rms}$, and $\rho_{i}>0$

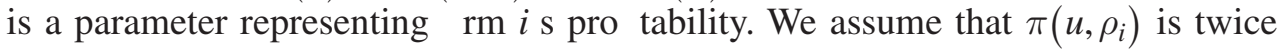

\footnotetext{
${ }^{27}$ In particular, $\underline{A} \equiv(1-\theta) \sqrt{\left(\Psi_{1}-\Psi_{3}\right)\left(\Psi_{2}-\Psi_{3}\right)}$ and $\stackrel{\otimes}{A}=2(1-\theta)\left[\left(1 / \Psi_{1}\right)+\left(1 / \Psi_{2}\right)+\left(1 / \Psi_{3}\right)\right]^{-1}$.

${ }^{28}$ Speci冈cally, existence can be demonstrated for $\left(1 / \Psi_{1}\right)+\left(1 / \Psi_{2}\right)>1 / \Psi_{3}$. This condition also ensures that $\underline{A}<\stackrel{\otimes}{A}$ and that Assumption A is satis囚ed for all $A<A$.
} 
continuously differentiable and increasing in $\rho_{i}$ for all $u \geq u^{m}$, where $u^{m}$ maximizes $\pi\left(u, \rho_{i}\right)$ for any $\rho_{i}$.

COROLLARY 3: Suppose a sales equilibrium exists with $k^{*}<n$, and that firms have symmetric shares of nonshoppers, $\theta_{i}=(\theta / n) \forall i$. Ceteris paribus, the firms that use sales will be those with the highest profitability, $\rho_{i}$, if increases in $\rho_{i}$ raise per-consumer profits relatively more at higher rather than lower utility levels, $\pi_{\rho u}(u, \rho) \geq 0 \forall u>u^{m}$.

Corollary 3 suggests that the effects of a $\bigotimes$ rm $₫$ pro®tability, $\rho_{i}$, on its use of sales are ambiguous. However, it predicts that more pro\table $₫$ rms are more likely to use sales if $\pi_{\rho u}(u, \rho) \geq 0 \forall u>u^{m}$. This condition is satis囚ed for several common situations. For instance, it applies under unit demand or two-part tariffs, where $\rho_{i}$ captures an increase in per-consumer demand or a reduction in marginal cost, and where $\pi_{\rho u}(\cdot)=0$ as $\pi_{\rho}(u, \rho)$ is independent of $u$. Thus, in these instances, $\nabla \mathrm{rms}$ with larger per-consumer demand or lower marginal costs should be more likely to use sales. Alternatively, it also applies under downward-sloping demand. ${ }^{29}$

\section{Applications}

Although our main contribution is methodological, this section®provides three applications to illustrate how our framework can be used to help future research. SectionXIIA examines a common procedure used in empirical work on sales and price dispersion. SectionХIIB considers a family of games where $\nabla r m s$ engage in a prior activity to in囚uence their share of nonshoppers, and Section囚IIC presents a number of comparative statics to further analyze the effects of $\nabla r m$ heterogeneities on sales.

\section{A. Implications for Empirical Procedures}

Within a given market, price dispersion is broadly divided into two forms. The Ørst $₫$ temporal $\bigotimes$ form involves price differences that vary over time, such as those generated by sales. The second $\bigotimes$ spatial $\bigotimes$ form arises from persistent inter $\bigotimes$ rm heterogeneities related to $₫ \mathrm{rms} \bigotimes$ characteristics. As listed in the introduction, many studies within the large empirical literature on sales attempt to focus on the temporal form by using a $₫$ cleaning $\bigotimes$ procedure. This procedure retrieves a set of price residuals from raw price data by using a regression involving observable $\nabla$ rm characteristics or $\bigotimes$ rm-level $₫ x e d$ effects. The price residuals are then interpreted as resulting from a homogeneous symmetric market and used to perform a reduced-form analysis or structural estimation. We now use our framework to better understand when this procedure is valid, and to suggest some modi囚cations for it to be applied more widely.

\footnotetext{
${ }^{29}$ Under downward-sloping demand with a minimum utility constraint, $u^{\min } \geq \min \left\{u_{i}^{m}, \ldots, u_{n}^{m}\right\}, \rho_{i}$ is best

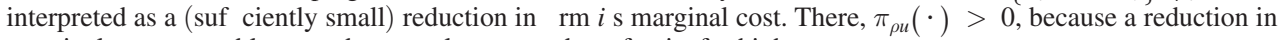
marginal cost pro®tably extends over a larger number of units for higher $u$.
} 
Wildenbeest (2011) provides the only formal justi冈cation for the cleaning procedure under a speci冈c set of market conditions. A version of his arguments can be derived in our framework, where in contrast, we generalize to positive advertising costs. Under unit demand and single products, suppose that rrms vary in product quality and costs subject to a common value-cost markup, $V_{i}-c_{i}=\Psi \forall i$. This implies symmetric pro $₫ \mathrm{t}$ functions, $\pi_{i}(u)=\Psi-u \forall i$. Hence, if $\nabla \mathrm{rms}$ also have symmetric shares of nonshoppers and advertising costs, then any sales equilibrium will involve symmetric utility distributions, $F_{i}(u)=F(u) \forall i$. Importantly, $\bigotimes$ rms $₫$ subsequent price distributions are then simple translations of each other as $p_{i}\left(u_{i}\right)=V_{i}-u_{i}$ under unit demand. Therefore, after observing a panel of price observations, one can obtain a measure of $\bigotimes$ rms \utility offers (under the assumption of a stationary, \nitely repeated game). Speci冈cally, one can regress the raw price data on a set of $\nabla \mathrm{rm}$-level $\nabla x \mathrm{xed}$ effects, $p_{i t}=\alpha+\delta_{i}+\varepsilon_{i t}$, to soak up the effects of the $\nabla r m$ heterogeneities and return a set of $\nabla$ cleaned $\nabla$ residuals that correctly proxy the utilities up to a positive constant. ${ }^{30}$

Downward-Sloping Demand.区 For the procedure to be valid under downward-sloping demand, one $\nabla$ rst needs a revised condition to ensure that $₫ \mathrm{rms} \bigotimes$ pro囚t functions are symmetric. While a more general condition can be provided, it is suf囚cient for our purposes to focus on the following simple case where each $\operatorname{\nabla rm} i$ has a marginal cost $c_{i} \geq 0$, and a linear per-consumer demand function that varies only in its intercept, $q_{i}\left(p_{i}\right)=a_{i}-$ $b p_{i}$ where $a_{i} \geq 0$ and $b>0$. From footnote 12 , we know $u_{i}=\left(a_{i}-b p_{i}\right)^{2} / 2 b$ and $\pi_{i}(u)=(1 / b)\left[a_{i}-b c_{i}-\sqrt{2 b u}\right][\sqrt{2 b u}]$. Hence, pro $\llbracket \mathrm{t}$ functions are symmetric if $a_{i}-b c_{i}=\Psi \forall i$. This restriction maintains some sense of Wildenbeest $\varangle$ constant value-cost assumption. Under this new condition, one would then aim to recover the \rms \utility draws from the raw price data. However, unlike unit demand, the relationship between prices and utilities is nonlinear, $u_{i}=\left(a_{i}-b p_{i}\right)^{2} / 2 b$. Therefore, the cleaning procedure no longer provides correct estimates of utility up to a positive constant. ${ }^{31}$ Instead, to recover the utility draws, one would have to use a more complex, data-intensive procedure to estimate some of the demand parameters.

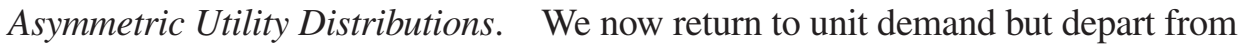
Wildenbeest $₫$ constant value-cost condition. Here, $\nabla$ rms $\bigotimes$ utility distributions will be asymmetric, $F_{i}(u) \neq F(u)$, with different mean utilities, $u_{i}^{\text {ave }}$, and so $\nabla \mathrm{rms} \bigotimes$ price distributions are no longer simple translations of each other. Hence, any $\nabla x e d-e f f e c t s$ regression cannot correctly proxy utilities up to a positive constant. Instead, one could

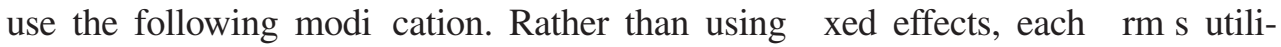
ties, $u_{i}=V_{i}-p_{i}$, could be estimated more directly from the price data by inferring $V_{i}$. As $\triangle \mathrm{rms}$ will set their highest price equal to $V_{i}$ with probability $\left(1-\alpha_{i}\right)>0, V_{i}$ can

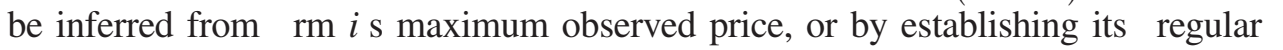

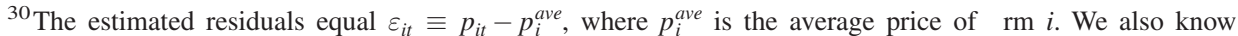
that $p_{i t}=V_{i}-u_{i t}$ and $p_{i}^{\text {ave }}=V_{i}-u^{\text {ave }}$, where $u^{\text {ave }}$ is the average utility from the symmetric utility distribution. Hence, $\mathbb{W}_{t} \equiv-\left(u_{i t}-u^{a v e}\right)$. For many applications, such as the estimation of search costs, this is suf囚cient as only the difference in utilities matters.

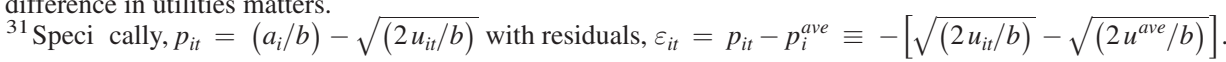


price using a statistical procedure (e.g., Hosken and Reiffen 2004). Having recovered the utilities, one could then use our framework to analyze the observed price dispersion or estimate a structural model. For instance, by using our theoretical predictions, one could use data on prices and advertising frequencies to estimate each $\nabla \mathrm{rm} \bigotimes$ share of nonshoppers, $\theta_{i}$, marginal cost, $c_{i}$, and/or advertising cost, $A_{i}$.

\section{B. Investment and Obfuscation Games}

We now illustrate how our framework capacity to allow for asymmetries can also help develop wider theoretical results. Speci®cally, we provide some results for a family of games where each $\nabla \mathrm{rm} \otimes$ share of nonshoppers is determined endogenously as a function of the $\triangle \mathrm{rms} \bigotimes$ actions prior to sales competition. Such actions can be interpreted as any (long-run) marketing activity that in囚uences consumer loyalty, such as (i) investments in persuasive advertising or sales force methods, or (ii) some forms of obfuscation that in囚uence the level of complexity or search costs within the market.

Starting with Chioveanu (2008), Carlin (2009), and Wilson (2010), some related streams of literature have become popular in recent years (see Grubb 2015 and Spiegler 2016 for reviews, and some wider related models). However, as explained in the introduction, only Baye and Morgan (2009) allow for positive costs of informative advertising, $A>0$; yet, due to the consequent dif囚culties of analyzing asymmetries, they only show the existence of a continuum of symmetric Nash equilibria and a unique symmetric equilibrium in secure strategies. In contrast, by utilizing the \exibility of our framework, we can characterize the unique symmetric subgame perfect Nash equilibrium (SPNE) across a general market setting (when it exists). Moreover, while their equilibria imply that an increase in the cost of informative advertising can have a positive, negative, or zero effect on the prior actions, we offer a unique, empirically testable prediction.

To begin, consider a market with $n \geq 2$ otherwise symmetric 冈rms. In stage 1, each $\nabla \mathrm{rm} i$ chooses its level of action, $e_{i} \geq 0$, with an associated unit cost equal

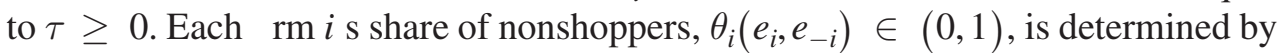
its own action, $e_{i}$, and the actions of its rivals, given by the vector $e_{-i}$. Furthermore, we assume $\theta_{i}\left(e, e_{-i}\right)=\theta_{j}\left(e, e_{-j}\right), \forall i, j$, whenever $e_{-j}$ is obtained from $e_{-i}$ by permutation. Then, in stage 2 , having observed the resulting shares, $\left\{\theta_{1}(\cdot), \ldots, \theta_{n}(\cdot)\right\}$, the rrms compete in utility with informative advertising costs, $A>0$, in line with our main model and under our previously stated conditions.

The exact form of the function, $\theta_{i}\left(e_{i}, e_{-i}\right)$, is allowed to depend upon the type of action. For instance, under $\mathbb{\Xi}($ own $)$ loyalty-increasing actions,$\bigotimes \theta_{i}\left(e_{i}, e_{-i}\right)$ is strictly

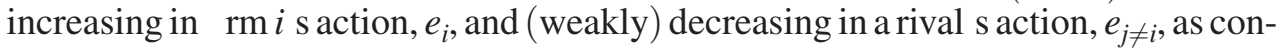
sistent with persuasive advertising. Alternatively, under $\mathbb{\nabla}$ (own) loyalty-decreasing actions $\unrhd \theta_{i}\left(e_{i}, e_{-i}\right)$ is strictly decreasing in $e_{i}$ and (weakly) increasing in $e_{j \neq i}$, as consistent with some forms of obfuscation. However, in either case, we assume that the total proportion of nonshoppers, $\theta(\cdot)=\sum_{i=1}^{n} \theta_{i}(\cdot)$, is inereasing and concave in any $\triangle \mathrm{rm} \bowtie$ action, $e_{i}$, with $\theta(\cdot) \rightarrow 1$ as $\sum_{i=1}^{n} e_{i}(\cdot) \rightarrow \infty .32$

\footnotetext{
${ }^{32}$ Hence, under a persuasive advertising interpretation, an increase in $e_{i}$ expands the total proportion of persuaded

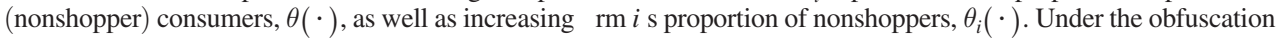


We now seek to characterize the SPNE of the two-stage game. In particular, we wish to characterize a symmetric SPNE where the $\nabla$ rms select positive actions before actively competing in a sales equilibrium (with $e_{i}=e>0$ and $\alpha_{i}=\alpha>0 \forall i$ ). To proceed, note that $\triangle \mathrm{rm} i$ will select its action, $e_{i}$, to maximize its equilibrium pro®ts (net of any direct action costs), $\Pi_{i}=\theta_{i}(\cdot) \pi^{m}+\left(x_{i}^{*} /\left(1-x_{i}^{*}\right)\right) A-\tau e_{i}$. However, for our current purposes, it is better to use an alternative expression based on the expected pro®ts from advertising the upper utility bound and attracting all the shoppers, $\prod_{i}=\pi(\rrbracket(\cdot))\left[1-\sum_{j \neq i} \theta_{j}(\cdot)\right]-A-\tau e_{i}$, where $\sharp(\cdot)$ is now a function of the $\nabla$ rms $₫$ actions. We can then state the following. 33

PROPOSITION 3: (i) When a symmetric SPNE exists with $e_{i}=e^{*}>0$ and $\alpha_{i}=\alpha^{*}>0 \forall i$, the unique level of action, $e^{*}$, satisfies the following FOC:

$$
\left.-\pi(\rrbracket(\cdot))(n-1) \frac{\partial \theta_{j}(\cdot)}{\partial e_{i}}+\left(1-\frac{(n-1)}{n} \theta(\cdot)\right) \pi^{\prime}(\stackrel{\boxplus}{\sharp} \cdot)\right) \frac{\partial 囚(\cdot)}{\partial e_{i}}-\tau=0
$$

(ii) When actions are own loyalty-increasing (or decreasing), each firm's equilibrium action, $e^{*}$, is strictly increasing (decreasing) in the costs of informative advertising, A.

Aside from increasing $\nabla \mathrm{rm}$ i 仗 total action costs by $\tau$, the FOC in (12) suggests that a marginal increase in $e_{i}$ generates two effects on the pro®ts of $\nabla \mathrm{rm} i$. The \rst effect changes the sum of available consumers that $\nabla \mathrm{rm} i$ can attract with a given offer of $\mathbb{X}$ by in囚uencing its $(n-1)$ rivals $\bigotimes$ shares of nonshoppers. Under own loyalty-increasing actions, this effect is (weakly) positive by reducing rivals $\nabla$ nonshoppers, but under own loyalty-decreasing actions, the effect is (weakly) negative. In contrast, the second effect is always positive for either form of action.

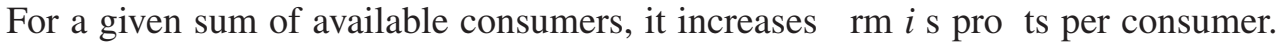
Speci凶cally, an increase in $e_{i}$ softens competition by raising the total proportion of nonshoppers, $\theta(\cdot)$, which prompts a reduction in the equilibrium value of $\mathbb{a}$, such that $\pi^{\prime}(\ddot{\sharp})\left(\partial \rrbracket / \partial e_{i}\right)>0$.

Now consider the comparative statics. An increase in the costs of informative advertising, $A$, enhances both of the main effects in (12). Within the $\nabla r s t$ effect, an increase in $A$ lowers $₫$ by reducing the $\nabla$ rms $\bigotimes$ incentives to use advertised sales. This enhances $\bigotimes \mathrm{rm} i \mathbb{\otimes}$ reward from attracting available consumers, $\pi(\sharp)$. Thus, to reduce rival nonshopper shares, $\triangle \mathrm{rm} i$ will wish to select a higher action under own loyalty-increasing investment, and a lower action under own loyalty-decreasing investment. Within the second effect, an increase in $A$ always enhances the incentives for $\nabla r m i$ to select a higher action by increasing the ability of actions to soften competition; a higher $A$ makes $见$ more sensitive to $e_{i}$.

interpretation, an increase in $e_{i}$ reduces $\nabla \mathrm{rm} i$ proportion of nonshoppers, $\theta_{i}(\cdot)$. However, the increase in $e_{i}$ also makes the rms harder to compare and so it also increases the total proportion of nonshoppers, $\theta(\cdot)$.

${ }^{33}$ While Proposition 3 demonstrates uniqueness, equilibrium existence remains dif囚cult to verify in the general case. However, it is clear that the costs of informative advertising, $A$, must be moderate. If $A$ is suf $\bigotimes$ ciently small, no

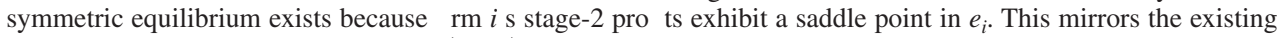
literature with $A=0$, e.g., Chioveanu (2008). However, if $A$ is too large, then no sales equilibrium will exist. 
Thus, under own loyalty-increasing actions both positive effects become larger, and so increases in the costs of informative advertising prompt higher equilibrium actions, $\partial e / \partial A>0$. Hence, reductions in the costs of informative advertising may be bene区cial to markets for two reasons: a direct effect in increasing sales competition, but also an indirect effect in reducing brand loyalty and thereby further reducing prices. To our knowledge, this result offers the $\nabla r s t$ theoretical prediction about how the costs of informative advertising affect equilibrium levels of loyalty-enhancing marketing activities such as persuasive advertising. It remains untested empirically.

Under own loyalty-decreasing actions, increases in $A$ prompt the $\nabla$ rst effect to become more negative and the second effect to become more positive. However, the \rst effect dominates such that increases in $A$ lower equilibrium actions, $\partial e / \partial A<0$. Hence, under an obfuscation interpretation, our model predicts that obfuscation should increase in response to reductions in informative advertising costs. This complements several theories that explain how \rms囚obfuscation levels should rise after a fall in search costs due to advances in search technologies (e.g., Ellison and Ellison 2009, Ellison and Wolitzky 2012).

\section{Comparative Statics}

This subsection®provides some new comparative static results. For symmetric market cases, the nndings extend standard clearinghouse results to a generalized market setting. More substantially, for asymmetric market cases where the existing literature has offered limited results, we offer several new insights by isolating the effects of individual $\nabla r m$ characteristics on sales and market performance. In line with footnote 8 , these predictions remain untested empirically, as the empirical literature has focused on other issues. For simplicity, we derive the statics under duopoly. However, related results can also be derived for $n>2$.

Changes in a Firm's Share of Nonshoppers. $\bigotimes$ Under symmetry, our framework

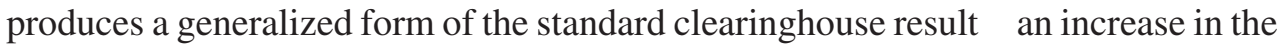
proportion of nonshoppers, $\theta$, (and associated reduction in shoppers, $1-\theta$ ) leads to a lower sales probability, $\alpha$, higher equilibrium pro®ts, $\Pi$, and lower average offers, $E(u)$. More interestingly, we can analyze a change in an individual $\nabla \mathrm{rm} \mathbb{B}$ share of nonshoppers, $\theta_{i}$. As these effects are dif囚cult to characterize, we focus on evaluating a small increase in $\theta_{i}$ at the point of symmetry. To proceed, one must also stipulate whether the increase is associated with a reduction in shoppers, $1-$ $\left(\theta_{i}+\theta_{j}\right)$, or rival nonshoppers, $\theta_{j}=\theta-\theta_{i}$. We 冈rst consider the latter.

PROPOSITION 4: In an otherwise symmetric market, consider a small increase in firm i's nonshoppers $\theta_{i}$ (and associated reduction in $\theta_{j}$ ). Starting from a point of symmetry, $\theta_{i}=\theta_{j}$, this increases firm i's equilibrium profits, $\bar{\Pi}_{i}$, decreases firm $i$ 's sales probability, $\alpha_{i}$, and average offer, $E\left(u_{i}\right)$, and generates the opposite effects on firm j.

Ceteris paribus, an increase in $\theta_{i}$ reduces $\tilde{u}_{i}$ and makes $\bigotimes \mathrm{rm} i$ less willing to offer higher utilities. However, to maintain the $\nabla$ rms $\bigotimes$ incentives to employ a common $\mathbb{\|}$ 
in equilibrium, this is partially offset by a reduction in $\nabla r m$ i equilibrium tiebreak probability, $x_{i}^{*}$ (and an associated increase in $x_{j}^{*}$ ). Hence, when combined, these effects lead $\bigotimes \mathrm{rm} i(\bigotimes \mathrm{rm} j)$ to gain higher (lower) equilibrium pro囚ts, use sales with a lower (higher) probability, and set lower (higher) average utility offers. While intuitive, the last result about average utility offers differs from Arnold et l. (2011) which considers asymmetric $\theta_{i}$ with unit demand and $A>0$ under the past literature tiebreak rule. Instead, they suggest an increase in $\theta_{i}$ leads $\nabla \mathrm{rm} i$ to become more aggressive in its advertised prices and so offer higher average utility offers. Unlike our results, this $\bigotimes$ nding con冈icts with standard results under $A=0$ such as Narasimhan (1988). ${ }^{34}$

Changes in a Firm's Advertising Costs.凶 Under symmetry, one can verify a generalized form of the standard result $\nabla$ an increase in advertising costs, $A$, leads to a lower sales probability, $\alpha$, higher equilibrium pro®ts, $П$, and lower average offers, $E(u)$. More substantially, our framework can now isolate the effects from a change in an individual $\nabla r m \varangle$ advertising cost, $A_{i}$.

PROPOSITION 5: In an otherwise symmetric market, a small increase in firm i's advertising cost, $A_{i}$, leads to no change in firm i's equilibrium profits, $\bar{\Pi}_{i}$, an increase in firm j's equilibrium profits, $\bar{\Pi}_{j}$, and a decrease in both firms' sales probabilities and average offers, $\alpha_{k}$ and $E\left(u_{k}\right)$ for $k=i, j$.

Ceteris paribus, an increase in $A_{i}$ reduces $\tilde{u}_{i}$ and makes $\nabla \mathrm{rm} i$ less willing to offer higher utilities. However, to maintain a common $\mathbb{x}$, this is also accompanied by a reduction in $\nabla \mathrm{rm}$ i 仗 equilibrium tiebreak probability, $x_{i}^{*}$, (and associated increase in $x_{j}^{*}$ ). This leads to no aggregate effect on $\prod_{i}$ because the direct effect from $A_{i}$ is exactly offset by the indirect effect from $x_{i}^{*}$. However, an increase in $A_{i}$ raises $\nabla \mathrm{rm} j \mathbb{}$ 『 pro®ts, $\prod_{j}$, because the indirect effect raises $x_{j}^{*}$. Hence, given our assumptions, the standard pro®t result under symmetry is driven by the rise in rival, rather than own, advertising costs. Finally, the increase in $A_{i}$ reduces both $\nabla \mathrm{rms} \bigotimes$ use of sales and prompts a subsequent reduction in their average offers.

Changes in a Firm's Profit Function.囚 As previously explained, studying variations in $\triangle \mathrm{rms} \bigotimes$ pro冈t functions is dif囚cult at a general level. However, we now provide some comparative statics by using the form of pro冈t function introduced in SectionХID, $\pi(u, \rho)$. In particular, we focus on situations where an increase in

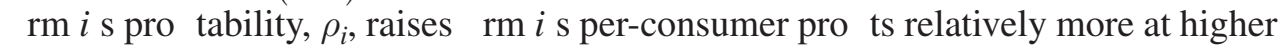
rather than lower utility levels, $\pi_{\rho u}(u, \rho) \geq 0 \forall u>u^{m}$, which we argued was consistent with many market environments.

Under symmetry, one can derive a new result; an industry-wide increase in prof-

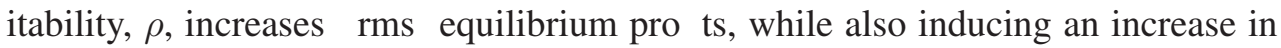

\footnotetext{
${ }^{34}$ With two exceptions, our $\otimes$ ndings remain robust in the alternative case where the increase in $\theta_{i}$ comes from a reduction in shoppers. First, an increase in $\theta_{i}$ now raises $\Pi_{j}$ because there is no reduction in $\theta_{j}$. Second, an increase in $\theta_{i}$ can provide reversed effects on $\alpha_{j}$ and $E\left(u_{j}\right)$ if advertising costs are relatively high. This arises due to the opposing effects of a decrease in shoppers, and an increase in $x_{j}^{*}$ (which varies in $A$ ). However, $\operatorname{vrm} i$ still offers a lower average utility than $\bigotimes \mathrm{rm} j$.
} 
the probability of sales, $\alpha$, and average utility offers, $E(u)$. In addition, we can also isolate the effects from a change in an individual $\bigotimes$ rm $₫$ pro®tability, $\rho_{i}$. The existing literature has only been able to consider a few speci囚c cases involving individual changes in marginal costs or product values under unit demand and zero advertising costs (e.g., Kocas and Kiyak 2006). Related technical dif囚 framework. However, by evaluating a small change at the point of symmetry, we can substantially improve upon past results.

PROPOSITION 6: In an otherwise symmetric market, consider a small increase in firm i's profitability, $\rho_{i}$. Starting from a point of symmetry, $\rho_{i}=\rho_{j}$, this increases firm i's equilibrium profits, $\bar{\Pi}_{i}$, sales probability, $\alpha_{i}$, and average offer, $E\left(u_{i}\right)$, but decreases firm j's equilibrium profits, $\bar{\Pi}_{j}$.

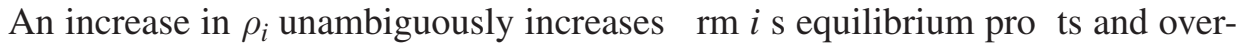
all industry pro®ts. Further, in the common cases where $\pi_{\rho u}(u, \rho) \geq 0$, an increase in $\rho_{i}$ also raises $\nabla$ rm $i \bigotimes$ incentive to advertise higher utilities. This prompts an increase in $\bigotimes \mathrm{rm}$ i邓 equilibrium tiebreak probability, $x_{i}^{*}$, to maintain a common $囚$. Nevertheless, $\nabla \mathrm{rm} i$ increases it sales probability, $\alpha_{i}$, and its average offer, $E\left(u_{i}\right)$, while $\bigotimes \mathrm{rm} j$ receives lower equilibrium pro囚ts, $\prod_{j \cdot}{ }^{35}$

\section{Conclusion}

Due to the associated technical complexities, existing clearinghouse sales models are unable to fully consider the effects of $\nabla r m$ heterogeneity. This restricts theoretical understanding, empirical analysis, and policy guidance with regards to sales and price dispersion, and many other topics in related literatures. The current paper has tried to $\bigotimes 11$ this gap by providing a generalized clearinghouse sales framework. In addition, the paper has (i) provided original insights into the number and types of \rms that use sales, (ii) offered new results on how $\nabla$ rm heterogeneity affects market outcomes, (iii) extended a $₫$ cleaning $\bigotimes$ procedure that is commonly used within the empirical literature, and (iv) analyzed a family of games to study persuasive advertising and obfuscation in sales markets. By opening up the analysis of sales with $\$ rm heterogeneity, we hope that our framework can enable future research to further address many theoretical and empirical issues that remain underexplored.

\section{A}

This appendix contains a number of equilibrium speci邓cations by further detailing the exact source of utility and pro囚t function. To help exposition, we illustrate the main steps within a context of full symmetry. Here, using the results from Sections \IC and IID, this also permits us to easily include the equilibrium details

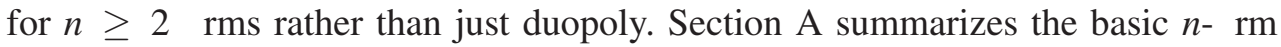
equilibrium under full symmetry. Sections $₫ \mathrm{~B} \bigotimes \mathrm{D}$ then detail the equilibrium under

\footnotetext{
${ }^{35}$ In general, the effects on $\alpha_{j}$ and $E\left(u_{j}\right)$ are more nuanced. However, for $\AA^{\bigotimes}$ suf囚ciently close to $u^{m}$, an increase in $\rho_{i}$ leads both $\otimes \mathrm{rms}$ to increase their sales probabilities and average offers.
} 
unit demand, downward-sloping demand, and multiple products, while discussing how our framework can reproduce and substantially extend many of the past literature $\$$ key predictions for pricing, advertising, and purchasing behavior. Sections $\mathbb{E} \mathbb{E} \mathrm{F}$ demonstrate how Proposition 1 can be used to characterize some common forms of sales that have remained unstudied within the clearinghouse literature, including cases where $\bigotimes$ rms use two-part tariffs or nonprice variables such as package size (e.g., $₫ X \%$ Free $\bigotimes)$.

\section{A. Equilibrium under Full Symmetry}

Using the results from Sections $\$$ IC and IID, we can now summarize the basic $n$ - $\nabla r m$ equilibrium under full symmetry. Here, all $\triangle$ rms will use sales in equilibrium and the equilibrium tiebreak rule will be fully symmetric, $x_{i}^{*}=1 / n$. In addition, for all $i$, we know $\AA_{i}=\frac{\theta \pi^{m}}{n}+\frac{A}{n-1}, \alpha_{i}=1-\left(\frac{\frac{n}{n-1} A}{(1-\theta) \pi^{m}}\right)^{\frac{1}{n-1}}$, 叉 $=\pi^{-1}\left(\frac{\theta \pi^{m}+\frac{n^{2}}{n-1} A}{\theta+n(1-\theta)}\right)$ and $F_{i}(u)=\left(\frac{\theta\left[\pi^{m}-\pi(u)\right]+\frac{n^{2}}{n-1} A}{n(1-\theta) \pi(u)}\right)^{\frac{1}{n-1}}$.

\section{B. Equilibrium with Unit Demand}

Building on footnote 12, suppose $u_{i}=V-p_{i}$ and $\pi\left(u_{i}\right)=V-c-u_{i}$, where $u_{i}^{m}=0$ and $\pi^{m}=V-c$. Under full symmetry, this produces a simple clearinghouse equilibrium with $x_{i}^{*}=1 / n, \quad \prod_{i}=\frac{\theta(V-c)}{n}+\frac{A}{n-1}$, $\alpha_{i}=1-\left(\frac{\frac{n}{n-1} A}{(1-\theta)(V-c)}\right)^{\frac{1}{n-1}}, \quad$ and $\quad \mathbb{x}=\frac{n(1-\theta)(V-c)-\frac{n^{2}}{n-1} A}{\theta+n(1-\theta)}$. By using $F_{i}(p)=1-F_{i}(u)$, one can further derive $F_{i}(p)=1-\left(\frac{\theta(V-p)+\frac{n^{2}}{n-1} A}{n(1-\theta)(p-c)}\right)^{\frac{1}{n-1}}$ with $p^{m}=V-u^{m}=V$ and $p=V-\mathbb{R}$. This collapses to the (popularized) equilibrium of Varian (1980) when $A \rightarrow 0$. Under \rm heterogeneity, the past literature has considered various asymmetries in nonshopper shares, product values, and/or costs in the duopoly setting under the restriction, $A_{i}=A_{j}=0$. As detailed in online Appendix $\mathrm{C} 1$, our framework can obtain these equilibria in the limit when $A_{i}=A_{j} \rightarrow 0$ while allowing for any $\theta_{i}, c_{i}$, and $V_{i}$. Moreover, our framework can also extend them to allow for positive and asymmetric advertising costs.

\section{Equilibrium with Downward-Sloping Demand}

Suppose each consumer has a downward-sloping demand function for $\nabla \mathrm{rm} i \mathbb{\varangle}$ good, $q_{i}\left(p_{i}\right)$, and that $\triangle \mathrm{rm} i$ has a constant marginal cost, $c_{i} \geq 0$. Firm $i$ then has a per-consumer pro囚t function equal to $\pi_{i}\left(p_{i}\right)=\left(p_{i}-c_{i}\right) q_{i}\left(p_{i}\right)$, and the utility at $\triangle \mathrm{rm} i$ can be given by its associated consumer surplus, $u_{i}=S\left(p_{i}, q_{i}\left(p_{i}\right)\right)$. Under our sales equilibrium, each $₫ \mathrm{rm} i$ then chooses its price to maximize its profits subject to supplying its required utility draw, $u_{i}$, with $p_{i}^{*}\left(u_{i}\right)=\arg \max _{p_{i}} \pi_{i}\left(p_{i}\right)$ subject to $S\left(p_{i}, q_{i}\left(p_{i}\right)\right)=u_{i}$. It also follows that $p_{i}^{m}=\arg \max _{p_{i}} \pi_{i}\left(p_{i}\right)$, with $u_{i}^{m}=S\left(p_{i}^{m}, q_{i}\left(p_{i}^{m}\right)\right)$ and $\pi_{i}^{m} \equiv \pi_{i}\left(u_{i}^{m}\right)=\pi_{i}\left(p_{i}^{m}\right)$. 
Under full symmetry, this produces a standard clearinghouse equilibrium (e.g. Baye, Morgan, and Scholten 2004a). Speci邓cally, it follows that $x_{i}^{*}=1 / n, \prod_{i}$ $=\frac{\theta \pi\left(p^{m}\right)}{n}+\frac{A}{n-1}, \alpha_{i}=1-\left(\frac{\frac{n}{n-1} A}{(1-\theta) \pi\left(p^{m}\right)}\right)^{\frac{1}{n-1}}$, and $\mathbb{\bigotimes}=\pi^{-1}\left(\frac{\theta \pi\left(p^{m}\right)+\frac{n^{2}}{n-1} A}{\theta+n(1-\theta)}\right)$. Using $F_{i}(p)=1-F_{i}(u)$, it follows that $F_{i}(p)=1-\left(\frac{\theta\left[\pi\left(p^{m}\right)-\pi(p)\right]+\frac{n^{2}}{n-1} A}{n(1-\theta) \pi(p)}\right)^{\frac{1}{n-1}}$ with $\underline{p}=p^{*}(\mathbb{囚})$ and $\stackrel{\bigotimes}{p}=p^{*}\left(u^{m}\right)=p^{m} \cdot{ }^{36}$ Our framework shows how this clearinghouse equilibrium can be generalized to allow for asymmetric advertising costs, nonshopper shares, and pro®t functions (provided there is some binding minimum utility constraint if needed).

\section{Equilibrium with Downward-Sloping Demand and Multiple Products}

An equilibrium with downward-sloping demand where $\nabla$ rms sell multiple products can be derived as an extension of Section $\mathrm{C}$ above. In particular, now suppose $\triangle \mathrm{rm} i$ has $K_{i} \geq 1$ products, where $\mathbf{c}_{i}=\left\{c_{i 1}, \ldots, c_{i K_{i}}\right\}, \mathbf{p}_{i}=\left\{p_{i 1}, \ldots, p_{i K_{i}}\right\}$, and $\mathbf{q}_{i}\left(\mathbf{p}_{i}\right)=\left\{q_{i 1}\left(\mathbf{p}_{i}\right), \ldots, q_{i K_{i}}\left(\mathbf{p}_{i}\right)\right\}$ denote the associated vectors of (constant) marginal costs, prices, and product demand functions per consumer. Many of the steps from Section $\mathbb{C}$, then, apply immediately. In particular, under suitable demand assumptions, there exists a unique price vector that maximizes a $\nabla r m \varangle$ pro囚ts subject to supplying a given utility draw $u$ across its products, such that $\mathbf{p}_{i}^{*}(u)=\arg \max _{\mathbf{p}_{i}} \pi_{i}\left(\mathbf{p}_{i}\right)=\mathbf{q}_{i}^{*}\left(\mathbf{p}_{i}\right)^{\prime}\left(\mathbf{p}_{i}-\mathbf{c}_{i}\right)$ subject to $S\left(\mathbf{p}_{i}, \mathbf{q}_{i}^{*}(\mathbf{p})\right)=u$, with resulting pro囚ts per consumer, $\pi_{i}(u)=\pi_{i}\left(\mathbf{p}_{i}^{*}(\mathbf{u})\right) \cdot{ }^{37}$ Under full symmetry, this reproduces versions the equilibrium of Simester (1997) when marginal costs are zero, $K \geq 1$, and $A \rightarrow 0$. More substantially, for any marginal costs and any $K$, we can permit positive asymmetric advertising costs and asymmetric shares of nonshoppers.

\section{E. Equilibrium with Two-Part Tariffs}

In line with the associated discussion in SectionХIC, suppose the $\nabla r m s$ employ two-part tariffs to better extract consumer surplus as consistent with a form of oligopolistic 冈rst-degree price discrimination. In particular, reconsider the analysis in Sections $\mathrm{C}$ and $\mathrm{D}$ above where $\operatorname{\nabla rm} i$ has $K_{i} \geq 1$ products, downward-sloping demand functions, $\mathbf{q}_{i}(\cdot)$, and marginal costs, $\mathbf{c}_{i}$. However, now let each $\operatorname{\nabla rm} i$ set a $K_{i}$-dimensional vector of marginal prices (per unit of consumption), $\mathbf{p}_{i}$, and a single $\nabla$ xed fee, $f_{i} \geq 0$. It then follows that $\pi_{i}\left(\mathbf{p}_{i}, f_{i}\right)=\mathbf{q}_{i}\left(\mathbf{p}_{i}\right)^{\prime}\left(\mathbf{p}_{i}-\mathbf{c}_{i}\right)+f_{i}$ and $u_{i}=S\left(\mathbf{p}_{i}, \mathbf{q}_{i}\left(\mathbf{p}_{i}\right)\right)-f_{i}$ where $S\left(\mathbf{p}_{i}, \mathbf{q}_{i}\left(\mathbf{p}_{i}\right)\right)$ denotes a consumer $\$$ surplus at $i$ gross of $i \varangle$ खxed fee. To generate any utility, $u^{\prime}, i$ will choose $\mathbf{p}_{i}$ and $f_{i}$ to maximize $\pi_{i}\left(\mathbf{p}_{i}, f_{i}\right)$ subject to $S\left(\mathbf{p}_{i}, \mathbf{q}_{i}\left(\mathbf{p}_{i}\right)\right)-f_{i}=u^{\prime}$. This implies marginal cost

\footnotetext{
${ }^{36}$ For comparison, standard clearinghouse models express the price distribution conditional on advertising. In our model, this equates to $F_{i}^{A}(p)=\left(1-F_{i}(u)\right) / \alpha_{i}$.

${ }^{37}$ This constrained pricing decision can be thought of as a Ramsey problem. Individual prices are hard to fully characterize, but with additional restrictions, \rms can be shown to optimally use lower prices on products that are more price-elastic and complementary to other products. See Simester (1997) and Armstrong and囚Vickers (2001) for more discussion.
} 
pricing across each product, $\mathbf{p}_{i}=\mathbf{c}_{i}$, together with a suitably adjusted $\nabla x e d$ fee, $f_{i}=S\left(\mathbf{c}_{i}, \mathbf{q}_{i}\left(\mathbf{c}_{i}\right)\right)-u^{\prime}$. The full equilibrium can then be derived using $\pi_{i}(u)=S\left(\mathbf{c}_{i}, \mathbf{q}_{i}\left(\mathbf{c}_{i}\right)\right)-u, u^{m}=0$, and $\pi_{i}\left(u^{m}\right)=S\left(\mathbf{c}_{i}, \mathbf{q}_{i}\left(\mathbf{c}_{i}\right)\right)$.

\section{F. Equilibrium with Nonprice Sales}

For ease of exposition, consider a fully symmetric market with single products and unit demand. In line with the associated discussion in SectionХIC, suppose each $\nabla \mathrm{rm}$ price is $\nabla x \mathrm{xed}$ at $p>0$, and that each -rm employs some other sales variable $z_{i} \in[\underline{z}, \underset{l}{\Omega}$. To avoid any unnecessary complications, we make two assumptions to ensure unique correspondences between $z_{i}, u_{i}$, and $\pi\left(u_{i}\right)$. First, let

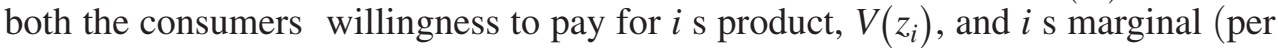
unit) cost, $c\left(z_{i}\right)$, be strictly increasing in $z_{i}$, such that $u\left(z_{i}\right)=V\left(z_{i}\right)-p$ is strictly increasing in $z_{i}$, and $\pi\left(z_{i}\right)=p-c\left(z_{i}\right)$ is strictly decreasing in $z_{i}$. Second, let

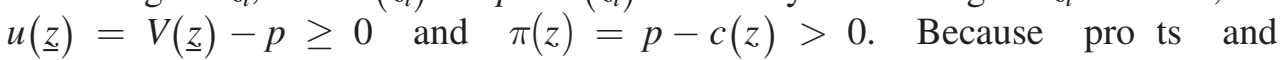
utilities are monotone in $z$, we have $z=V^{-1}(p+u)$. We can then derive $\pi(u)=p-c\left(V^{-1}(p+u)\right)$ and $u^{m}=V(\underline{z})-p$. To ensure the equilibrium exists, we can verify that $\pi\left(u^{m}\right)=p-c(\underline{z})>0$ and $\pi^{\prime}(u)<0$. The full equilibrium can then be explicitly derived and shown to exhibit the features listed in Section》IC.

Appendices B and C can be found in the supplementary online Appendix.

\section{REFERENCES}

Allen, Jason, Robert Clark, and Jean-François Houde. 2014. 『The Effect of Mergers in Search Markets: Evidence from the Canadian Mortgage Industry $\bigotimes$ American Economic Review 104 (10): 3365区96.

An, Yonghong, Michael R. Baye, Yingyao Hu, John Morgan, and Matt Shum. 2017. 『ldenti邓cation and Estimation of Online Price Competition with an Unknown Number of Firms \ Journal of Applied Econometrics 32 (1): 80冈102.

Anderson, Simon P., Alicia Baik, and Nathan Larson. 2015. 区Personalized Pricing and Advertising: An Asymmetric Equilibrium Analysis \ Games and Economic Behavior 92: 53囚73.

Anderson, Simon P., and Régis Renault. 2018. 『Firm Pricing with Consumer Search. In Handbook of Game Theory and Industrial Organization, Vol. 2, edited by Luis C. Corch冈n and Marco A. Marini, 177区224. Northampton, MA: Edward Elgar.

Armstrong, Mark. 2015. 凶Search and Ripoff Externalities $\bigotimes$ Review of Industrial Organization 47 (3): 273囚302.

Armstrong, Mark, and John Vickers. 2001. \Competitive Price Discrimination $\bigotimes$ RAND Journal of Economics 32 (4): 579冈605.

Armstrong, Mark, and John Vickers. 2019. 凶Discriminating against Captive Customers $\bigotimes$ American Economic Review: Insights 1 (3): 257囚72.

-Arnold, Michael, Chenguang Li, Christine Saliba, and Lan Zhang. 2011. XAsymmetric Market Shares, Advertising and Pricing: Equilibrium with an Information Gatekeeper.囚 Journal of Industrial Economics 59 (1): 63区84.

Barron, John M., Beck A. Taylor, and John R. Umbeck. 2004. 囚Number of Sellers, Average Prices, and Price Dispersion.凶International Journal of Industrial Organization 22 (8区9): 1041 66.

-Baye, Michael R., Dan Kovenock, and Casper G. de Vries. 1992. 区lt Takes Two to Tango: Equilibria in a Model of Sales.凶 Games and Economic Behavior 4 (4): 493囚510.

Baye, Michael R., and John Morgan. 2001. 囚lnformation Gatekeepers on the Internet and the Competitiveness of Homogeneous Product Markets. \American Economic Review 91 (3): $454 \bigotimes 74$.

Baye, Michael R., and John Morgan. 2009. 凶Brand and Price Advertising in Online Markets. $\$ Management Science 55 (7): 1139冈51. 
- Baye, Michael R., John Morgan, and Patrick Scholten. 2004a. 区Price Dispersion in the Small and in the Large: Evidence from an Internet Price Comparison Site $\bigotimes$ Journal of Industrial Economics 52 (4): 463凶96.

Baye, Michael R., John Morgan, and Patrick Scholten. 2004b. ХTemporal Price Dispersion: Evidence from an Online Consumer Electronics Market\Journal of Interactive Marketing 18 (4): 101囚15.

Baye, Michael R., John Morgan, and Patrick Scholten. 2006. XInformation, Search, and Price Disper-

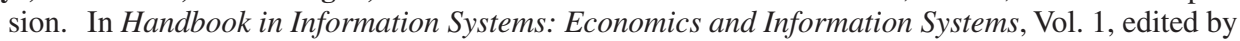
Terrence Hendershott, 323区75. Bingley, UK: Emerald Group Publishing Limited.

Brown, Jeffrey R., and Austan Goolsbee. 2002. 凶Does the Internet Make Markets More Competitive?

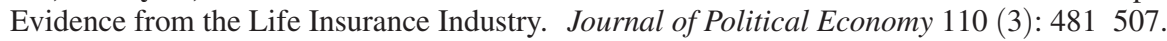

-Burdett, Kenneth, and Kenneth L. Judd. 1983. 『Equilibrium Price Dispersion.囚Econometrica 51 (4): 955凶69.

- Burdett, Kenneth, and Guido Menzio. 2018. 『The (Q, S, s) Pricing Rule. \Review of Economic Studies 85 (2): 892ख928.

-Carlin, Bruce I. 2009. 囚Strategic Price Complexity in Retail Financial Markets. $\$ Journal of Financial Economics 91 (3): $278 \llbracket 87$.

-Chandra, Ambarish, and Mariano Tappata. 2011. 『Consumer Search and Dynamic Price Dispersion: An Application to Gasoline Markets $₫ R A N D$ Journal of Economics 42 (4): 681囚704.

-Chen, Haipeng, Howard Marmorstein, Michael Tsiros, and Akshay R. Rao. 2012. खWhen More Is Less: The Impact of Base Value Neglect on Consumer Preferences for Bonus Packs over Price Discounts. $\$ Journal of Marketing 76 (4): $64 \rrbracket 77$.

-Chioveanu, Ioana. 2008. 区Advertising, Brand Loyalty and Pricing. \Games and Economic Behavior 64 (1): $68 \otimes 80$.

Dubovik, Andrei, and Maarten C.W. Janssen. 2012. 区Oligopolistic Competition in Price and Quality $\mathbb{\square}$ Games and Economic Behavior 75 (1): 120区38.

-Ellickson, Paul B., and Sanjog Misra. 2008. \Supermarket Pricing Strategies \Marketing Science 27 (5): 811区28.

-Ellison, Glenn, and Sara Fisher Ellison. 2009. खSearch, Obfuscation, and Price Elasticities on the Internet. $囚$ Econometrica 77 (2): 427凶52.

-Ellison, Glenn, and Alexander Wolitzky. 2012. 『A Search Cost Model of Obfuscation\RAND Journal of Economics 43 (3): 417ه41.

-Galeotti, Andrea, and José Luis Moraga-González. 2009. खPlatform Intermediation in a Market for Differentiated Products. $₫$ European Economic Review 53 (4): 417凶28.

- Giulietti, Monica, Michael Waterson, and Matthijs Wildenbeest. 2014. 区Estimation of Search Frictions in the British Electricity Market. Journal of Industrial Economics 62 (4): 555区90.

-Grubb, Michael D. 2015. 囚Failing to Choose the Best Price: Theory, Evidence, and Policy $\bigotimes$ Review of Industrial Organization 47 (3): 303区40.

-Guimaraes, Bernardo, and Kevin D. Sheedy. 2011. 凶Sales and Monetary Policy \American Economic Review 101 (2): 844冈76.

-Hendel, Igal, Alessandro Lizzeri, and Nikita Roketskiy. 2014. \Nonlinear Pricing of Storable Goods $\mathbb{\square}$ American Economic Journal: Microeconomics 6 (3): 1囚34.

Hitsch, Günter J., Ali Hortacsu, and Xiliang Lin. 2019. खPrices and Promotions in U.S. Retail Markets: Evidence from Big Data. $\triangle$ Chicago Booth Working Paper 17-18.

Hosken, Daniel, and David Reiffen. 2004. खPatterns of Retail Price Variation $\bigotimes$ RAND Journal of Economics 35 (1): $128 \rrbracket 46$.

Hosken, Daniel, and David Reiffen. 2007. खPricing Behavior of Multiproduct Retailers $\bigotimes$ B.E. Journal of Theoretical Economics 7 (1): 1354凶54.

-Janssen, Maarten C.W., and José Luis Moraga-González. 2004. 囚Strategic Pricing, Consumer Search and the Number of Firms. \Review of Economic Studies 71 (4): 1089凶1118.

-Jensen, Robert. 2007. QThe Digital Provide: Information (Technology), Market Performance, and

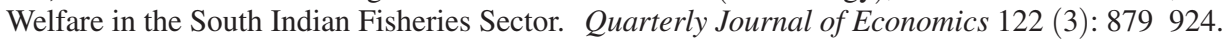

-Kaplan, Greg, and Guido Menzio. 2015. \The Morphology of Price Dispersion\ International Economic Review 56 (4): 1165区1206.

-Kaplan, Greg, and Guido Menzio. 2016. 『Shopping Externalities and Self-Ful冈lling Unemployment Fluctuations》 Journal of Political Economy 124 (3): 771ه825.

-Kocas, Cenk, and Tunga Kiyak. 2006. \Theory and Evidence on Pricing by Asymmetric Oligopolies $\mathbb{\square}$ International Journal of Industrial Organization 24 (1): 83凶105.

Lach, Saul. 2002. 区Existence and Persistence of Price Dispersion: An Empirical Analysis. $\triangle$ Review of Economics and Statistics 84 (3): 433区44. 
-Lach, Saul, and José L. Moraga-González. 2017. 『Asymmetric Price Effects of Competition囚 Journal of Industrial Economics 65 (4): 767ه803.

-Lewis, Matthew. 2008. 『Price Dispersion and Competition with Differentiated Sellers. $\bigotimes$ Journal of Industrial Economics 56 (3): 654囚78.

-Moraga-González, José Luis, Zsolt Sándor, and Matthijs R. Wildenbeest. 2013. खSemi-nonparametric Estimation of Consumer Search Costs.凶Journal of Applied Econometrics 28 (7): $1205 \rrbracket 23$.

Moraga-González, José Luis, and Matthijs R. Wildenbeest. 2012. 『Comparison Sites.凶In The Oxford Handbook of the Digital Economy, edited by Martin Peitz and Joel Waldfogel, 224囚53. New York: Oxford University Press.

Nakamura, Emi, and Jón Steinsson. 2008. 区Five Facts about Prices: A Reevaluation of Menu Cost Models $\bigotimes$ Quarterly Journal of Economics 123 (4): 1415囚64.

- Nakamura, Emi, Jón Steinsson, Patrick Sun, and Daniel Villar. 2018. \The Elusive Costs of In囚ation: Price Dispersion during the U.S. Great In囚ation》 Quarterly Journal of Economics 133 (4): $1933 \bigotimes 80$

-Narasimhan, Chakravarthi. 1988. 『Competitive Promotional Strategies \Journal of Business 61 (4): 427凶49.

-Palazon, Mariola, and Elena Delgado-Ballester. 2009. 『Effectiveness of Price Discounts and Premium Promotions. \Psychology and Marketing 26 (12): 1108囚29.

-Pennerstorfer, Dieter, Philipp Schmidt-Dengler, Nicolas Schutz, Christoph Weiss, and Biliana Yontcheva. 2020. XInformation and Price Dispersion: Theory and Evidence区 International Economic Review 61 (2): 871ه99.

Potters, Jan, and Sigrid Suetens. 2013. 『Oligopoly Experiments in the Current Millennium \Journal of Economic Surveys 27 (3): 439凶60.

-Robert, Jacques, and Dale O. Stahl, II. 1993. đInformative Price Advertising in a Sequential Search Model囚Econometrica 61 (3): 657凶86.

Ronayne, David. 2020. खPrice Comparison Websites $\bigotimes$ Warwick Economics Research Papers 1056.

-Shankar, Venkatesh, and Ruth N. Bolton. 2004. 区An Empirical Analysis of Determinants of Retailer Pricing Strategy. $\bigotimes$ Marketing Science 23 (1): 28区49.

-Shelegia, Sandro. 2012. 『Asymmetric Marginal Costs in Search Models \Economics Letters 116 (3): $551 \otimes 53$.

-Sherman, Joshua, and Avi Weiss. 2017. 『On Fruitful and Futile Tests of the Relationship between Search and Price Dispersion. $囚$ Economic Inquiry 55 (4): 1898区1918.

-Simester, Duncan. 1997. 区Note. Optimal Promotion Strategies: A Demand-Sided Characterization $\mathbb{Z}$ Management Science 43 (2): 251区56.

-Simon, Leo K., and William R. Zame. 1990. 『Discontinuous Games and Endogenous Sharing Rules $\rrbracket$ Econometrica 58 (4): 861区72.

-Sorensen, Alan T. 2000. 区Equilibrium Price Dispersion in Retail Markets for Prescription Drugs $\mathbb{X}$ Journal of Political Economy 108 (4): 833区50.

-Spiegler, Ran. 2016. 『Choice Complexity and Market Competition『Annual Review of Economics 8: $1 \otimes 25$.

Stahl, Dale O., II. 1989. 凶Oligopolistic Pricing with Sequential Consumer Search.凶American Economic Review 79 (4): 700ख12.

Varian, Hal R. 1980. 『A Model of Sales \American Economic Review 70 (4): $651 \otimes 59$.

-Wildenbeest, Matthijs R. 2011. 区An Empirical Model of Search with Vertically Differentiated Products $\triangle R A N D$ Journal of Economics 42 (4): 729凶57.

-Wilson, Chris M. 2010. \Ordered Search and Equilibrium Obfuscation. International Journal of Industrial Organization 28 (5): 496ญ506.

-Woodward, Susan E., and Robert E. Hall. 2012. 『Diagnosing Consumer Confusion and Sub-optimal Shopping Effort: Theory and Mortgage-Market Evidence \American Economic Review 102 (7): 3249凶76.

Zhang, Xing, Tat Y. Chan, and Ying Xie. 2018. 『Price Search and Periodic Price Discounts $\square$ Management Science 64 (2): 495区510. 